\title{
Practical Multiple Scattering for Rough Surfaces
}

\author{
JOO HO LEE, KAIST \\ ADRIAN JARABO, Universidad de Zaragoza, I3A \\ DANIEL S. JEON, KAIST \\ DIEGO GUTIERREZ, Universidad de Zaragoza, I3A \\ MIN H. KIM, KAIST
}
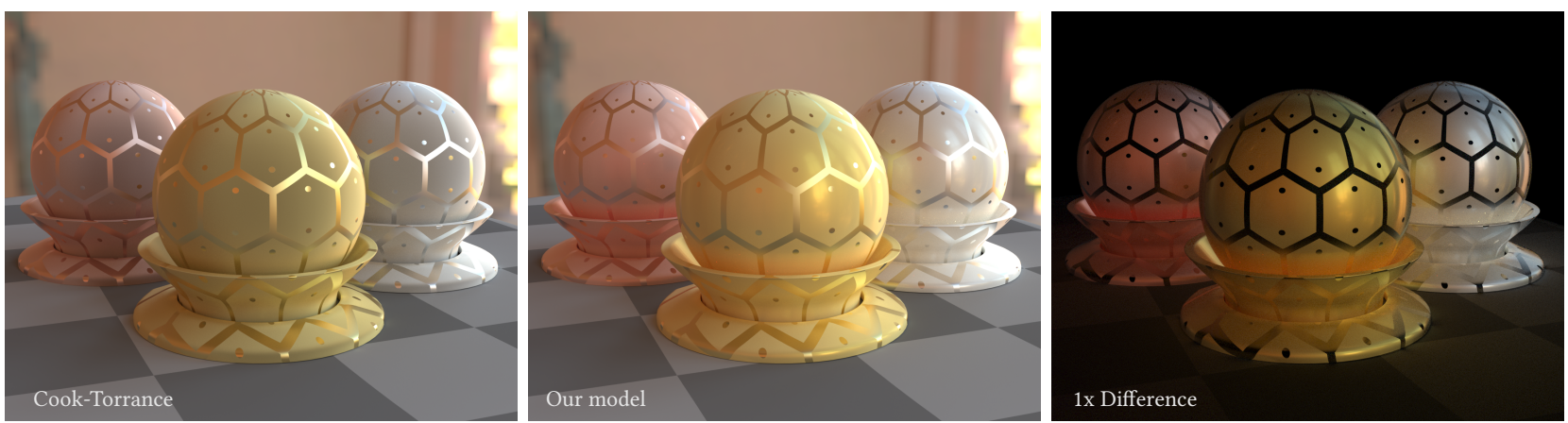

Fig. 1. Three different objects made of rough conductors (gold, silver, and copper), with spatially-varying roughness specified by a texture, rendered with the Cook-Torrance model (left) and our multiple-scattering-aware microfacet model (middle). Cook-Torrance assumes single scattering, which results in significant energy losses, specially for rough surfaces, as shown in the difference image (right). Our model accounts for multiple scattering in a closed-form analytical way, therefore conserving energy, with a small overhead, and without the need for costly stochastic light transport simulations.

Microfacet theory concisely models light transport over rough surfaces. Specular reflection is the result of single mirror reflections on each facet, while exact computation of multiple scattering is either neglected, or modeled using costly importance sampling techniques. Practical but accurate simulation of multiple scattering in microfacet theory thus remains an open challenge. In this work, we revisit the traditional V-groove cavity model and derive an analytical, cost-effective solution for multiple scattering in rough surfaces. Our kaleidoscopic model is made up of both real and virtual Vgrooves, and allows us to calculate higher-order scattering in the microfacets in an analytical fashion. We then extend our model to include nonsymmetric grooves, allowing for additional degrees of freedom on the surface geometry, improving multiple reflections at grazing angles with backward compatibility to traditional normal distribution functions. We validate the accuracy of our model against ground-truth Monte Carlo simulations, and demonstrate its flexibility on anisotropic and textured materials. Our model is analytical, does not introduce significant cost and variance, can be seamless integrated in any rendering engine, preserves reciprocity and energy conservation, and is suitable for bidirectional methods.

Authors' addresses: Joo Ho Lee, KAIST, School of Computing, Daejeon, South Korea 34141, jhlee@vclab.kaist.ac.kr; Adrian Jarabo, Universidad de Zaragoza, I3A, Zaragoza, Spain, 50018, ajarabo@unizar.es; Daniel S. Jeon, KAIST, School of Computing, Daejeon, South Korea, 34141, sjjeon@vclab.kaist.ac.kr; Diego Gutierrez, Universidad de Zaragoza, I3A, Zaragoza, Spain, 50018, diegog@unizar.es; Min H. Kim, KAIST, School of Computing, Daejeon, South Korea, 34141, minhkim@kaist.ac.kr(corresponding-author).

Permission to make digital or hard copies of all or part of this work for personal or classroom use is granted without fee provided that copies are not made or distributed for profit or commercial advantage and that copies bear this notice and the full citation on the first page. Copyrights for components of this work owned by others than ACM must be honored. Abstracting with credit is permitted. To copy otherwise, or republish to post on servers or to redistribute to lists, requires prior specific permission and/or a fee. Request permissions from permissions@acm.org.

(c) 2018 Association for Computing Machinery.

0730-0301/2018/11-ART275 \$15.00

https://doi.org/10.1145/3272127.3275016
CCS Concepts: • Computing methodologies $\rightarrow$ Reflectance modeling;

Additional Key Words and Phrases: microfacets, multiple scattering

ACM Reference Format:

Joo Ho Lee, Adrian Jarabo, Daniel S. Jeon, Diego Gutierrez, and Min H. Kim. 2018. Practical Multiple Scattering for Rough Surfaces. ACM Trans. Graph. 37, 6, Article 275 (November 2018), 12 pages. https://doi.org/10.1145/3272127. 3275016

\section{INTRODUCTION}

Microfacet theory [Beckmann and Spizzichino 1963; Torrance and Sparrow 1967] is one of the cornerstones of appearance modeling of real-world objects. At the macroscopic level, the theory postulates that the reflectance field at a surface point is the result of light reflections off several microscale specular facets with random orientations, defined by a statistical distribution function.

At the core of microfacet theory lies the assumption that light scatters on the microsurface only once (blue rays in Figure 2). This is adequate, since simplifies the computations. Unfortunately, it introduces energy loss, since multiple scattering inside the microsurface is ignored (red rays). A

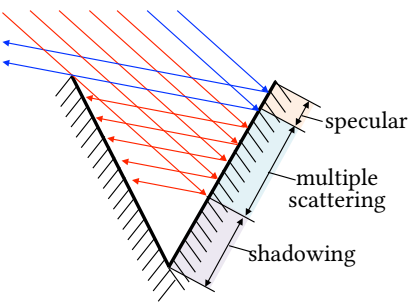

Fig. 2. Scattering in a V-groove cavity. common approach to compensate this is to introduce a Lambertian term that, under the assumption that multiple scattering inside the microsurface is diffuse, empirically brings back the lost energy. 
This in essence introduces a strong assumption: Microfacets, which are defined as perfectly specular, reflect light diffusely after the first bounce. Therefore, they present two different types of reflection, which is physically inaccurate, and might be problematic for instance when dealing with general anisotropic distributions of normals, as shown by Kulla and Conty [2017].

Recently, several efforts have been made to incorporate multiple scattering more accurately into microfacet theory, by formulating it as volumetric scattering [Dupuy et al. 2016; Heitz et al. 2016] relying on a Smith microfacet model [Smith 1967]. While this model is general, it requires a numerical stochastic evaluation of multiple scattering by tracing multiple random walks inside the microsurface. This is computationally expensive, requires non-trivial modifications of existing rendering systems, and introduces an additional source of variance.

In this paper, we develop a closed-form, analytical microfacet model that takes into account multiple scattering without a significant increase in computation. We analyze light transport inside a traditional V-groove cavity model [Cook and Torrance 1982], and develop an abstract description of multiple scattering inside a V-groove (which we name the kaleidoscopic V-groove model), which allows us to compute multiple scattering analytically, without expensive stochastic sampling methods.

In addition, we introduce a nonsymmetric variation of the model, which enables additional degrees of freedom on the surface's representation, removing the limiting assumption of symmetric Vgrooves in previous works. This results in better reflections at grazing angles, and is compatible with a broader set of microfacets normal distribution functions (NDF), including non-zero-mean and backfacing (i.e., defined over the full sphere) NDFs. Such distributions are important when depicting metallic materials [Dong et al 2015]. Our model does not require additional sampling for compute multiple scattering, can be used with any arbitrary rendering technique without major modifications, conserves energy, and preserves reciprocity. On the other hand, the correlation of the $\mathrm{V}$-grooves in the traditional model results in specular highlights from multiple scattering, even for materials exhibiting near-diffuse appearance; modeling non-parallel grooves would avoid this.

In summary, we offer the following three main contributions in Sections 4 and 5:

- A kaleidoscopic geometry analysis of multiple specular reflections within the traditional V-groove cavity model.

- An extension to include arbitrary nonsymmetric V-grooves.

- A reflection model of multiple specular scattering with arbitrary normal distributions for both symmetric and nonsymmetric V-groove cavities.

\section{RELATED WORK}

Microfacet models. Since the seminal work of Torrance and Sparrow [1967], several works have investigated the use of microfacets for modeling appearance. Cook and Torrance [1982] introduced microfacets for modeling the appearance of rough surfaces in computer graphics, based on the assumption of a microsurface formed by a set of symmetric $\mathrm{V}$-grooves, following the original Torrance and Sparrow model. Smith [1967] proposed an uncorrelated microsurface model, where the facet normals are independent on their position, which is suitable for rendering random surfaces [Heitz 2014], e.g., the surface of the sea. Ashikmin et al. [2000] independently proposed a shadowing/masking term, analogous to Smith's model. Walter et al. [2007] generalized microfacet to transmission, by creating a microfacet model for rough refraction.

Dong et al. [2015] compared the use of microfacets against a more accurate wave-optics reflectance model for predicting metals, proposing a non-zero-mean NDF for rough metallic surfaces, while Nam et al. [2016] used microfacets to accurately model microscale reflectance from captured data.

Holzschuch and Pacanowski [2017] proposed to incorporate an additional diffraction-aware nanoscale reflectance for microfacets for improving the accuracy for fitting measured materials. Other works model the appearance of surfaces beyond assuming a collection of randomly oriented facets, such as microcylinders [Hapke 1963; Poulin and Fournier 1990]. Recently, the V-groove model has been used for acquiring material appearance [Baek et al. 2018; Nam et al. 2018]. However, none of these works account for light transport due to multiple scattering inside the microfacets, therefore failing to conserve energy.

Multiple scattering in microfacets. A small number of works have attempted to account for multiple scattering in microfacets. Oren and Nayar [1994] developed a BRDF model for Lambertian symmetric $\mathrm{V}$-grooves, taking into account two bounces of scattering within the groove. Later, Saint-Pierre et al. [2018] further analyzed multiple scattering in Lambertian grooves beyond the second bounce. Koenderink et al. [1999] proposed a closed-form analytical BRDF model for microsurfaces modeled as spherical dents. In contrast, our work focuses on well-known, accurate and efficient representation of microsurfaces.

Raymond et al. [2016] modeled light transport on arbitrarily shaped scratches, extending previous works on V- [Merillou et al. 2001] and U-shaped [Bosch et al. 2004] scratches, accounting for multiple scattering via tabulating $2 \mathrm{D}$ light transport simulation. Kelemen and Szirmay-Kalos [2001] compensated the loss of multiple scattering by employing a pseudo-diffuse term. Jakob et al. [2014] later extended Kelemen's pseudo-diffuse correction to dielectric and conductor materials.

Recently, Heitz et al. [2016] proposed a model based on Smith's; following previous analysis of the reflectance field in random surfaces in physics [Bourlier and Berginc 2004; Bourlier et al. 2002], they modeled multiple scattering as an stochastic volumetric light transport process. The results show a good agreement with brute force simulations, although they rely on costly random walk sampling: this introduces an additional source of variance, and more importantly, it is computationally very expensive. Dupuy et al. [2016] further analyzed the relationship between multiple scattering in the Smith model and volumetric light transport in anisotropic media. Our kaleidoscopic model is closely related to Zipin's reflection model to compute heat transfer in metallic symmetric V-grooves [Zipin 1966]. Different from that work, we develop both symmetric and nonsymmetric variants, which we leverage to propose an efficient, radiometrically correct, closed-form BRDF accounting for multiple 
scattering in nonsymmetric V-grooves, including correct Fresnel reflection. Our model does not require any expensive stochastic sampling method for numerical light transport simulations and is suitable for existing bidirectional methods, satisfying reciprocity and energy conservation.

\section{BACKGROUND}

Microfacet theory [Torrance and Sparrow 1967] assumes that the appearance of a surface at the macroscopic level is the result of the interaction of a set of randomly oriented, perfectly specular facets at the microscopic scale. The orientation of the facets is defined statistically as a distribution function $D(\mathbf{h})$, where $\mathbf{h}=\frac{\mathbf{i}+\mathbf{o}}{\|\mathbf{i}+\mathbf{o}\|}$ is the halfway vector to indicate the angle between between $\mathbf{i}$ and $\mathbf{o}$. Mirror reflection on a microfacet occurs when its normal $\mathbf{s}$ is the same as $\mathbf{h}$ Following this model, the BRDF of a surface is modeled as [Walter et al. 2007]

$$
f(\mathbf{i}, \mathbf{o} ; \mathbf{n})=\frac{F(\mathbf{i}, \mathbf{h}) D(\mathbf{h}) G(\mathbf{i}, \mathbf{o}, \mathbf{h})}{4|\mathbf{i} \cdot \mathbf{n}||\mathbf{o} \cdot \mathbf{n}|},
$$

where $\mathbf{n}$ is the geometric normal at the surface, $F(\mathbf{i}, \mathbf{h})$ is the Fresnel reflection term, and $G(\mathbf{i}, \mathbf{o}, \mathbf{h})$ is the geometric term, accounting for the shadowing and masking between microfacets, and preserving the projected area of the visible microfacets. Although several microsurface models have been proposed in computer graphics, the most popular ones are the Smith [1967] and the V-groove cavity [Cook and Torrance 1982; Torrance and Sparrow 1967] models. These two are mathematically well-defined, and can be derived from microfacet profiles [Heitz 2014].

In this work, we focus on the V-cavity model shown in Figure 2. It assumes that the surface is made up of symmetric grooves with aperture angle $\theta_{v}$ and bisector aligned with $\mathbf{n}$. This geometry imposes a strong correlation between facets, so that a facet can only be shadow-masked by its adjacent-facing facet. This results in a masking term given by

$$
G_{1}(\mathbf{o}, \mathbf{h})=H\left(\frac{\mathbf{o} \cdot \mathbf{h}}{\mathbf{o} \cdot \mathbf{n}}\right) \min \left(1,2 \frac{|\mathbf{h} \cdot \mathbf{n}||\mathbf{o} \cdot \mathbf{n}|}{|\mathbf{o} \cdot \mathbf{h}|}\right),
$$

where $H(\cdot)$ is the Heaviside function ensuring that backfacing microfacets are discarded. The $G_{1}$ function models the masked visible area of a V-groove with halfway vector $\mathbf{h}$ at direction $\mathbf{o}$. Then, the total energy lost by both masking and shadowing at directions $\mathbf{i}$ and o can be computed from the geometric term

$$
G(\mathbf{i}, \mathbf{o}, \mathbf{h})=\min \left(G_{1}(\mathbf{i}, \mathbf{h}), G_{1}(\mathbf{o}, \mathbf{h})\right) .
$$

This geometric term is very simple and efficient to compute, and is independent of the distribution of normals used.

\section{KALEIDOSCOPIC ANALYSIS OF MULTIPLE REFLECTIONS IN V-GROOVE}

The common approach to compute reflections inside a V-groove is to generate a new reflected ray defined in the frame of reference of the geometry. Instead, the key intuitive idea of our model is to formulate reflections from the frame of reference of the ray. From this perspective, multiple reflections can be described within a set of consecutive, virtual V-grooves (which forms our kaleidoscopic model) in an analytical way, by simply computing the line equation. In the rest of this section, we first introduce the geometry of our model

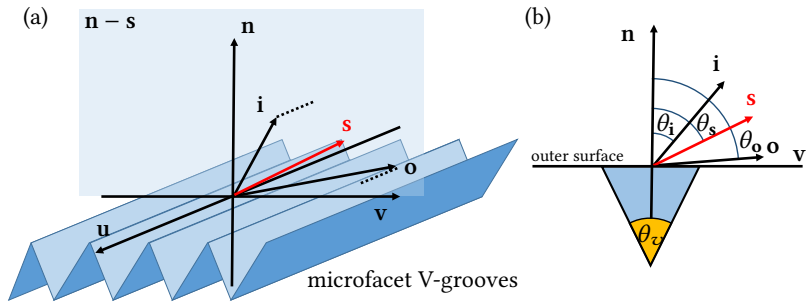

Fig. 3. Reference frame of our V-grooves. (a) Incident and exitant rays (i and o) are projected onto the $\mathbf{n}$-s plane (defined by the geometric normal $\mathbf{n}$ and the microfacet normal s), perpendicular to the direction of the groove (uaxis). (b) Given that the azimuth angle $\phi$ with respect to $\mathbf{n}-\mathbf{s}$ is not affected by reflection, we operate on the elevation angles $\theta_{\mathbf{i}}, \theta_{\mathbf{s}}$, and $\theta_{\mathbf{o}}$ (for $\mathbf{i}, \mathbf{s}$, and o respectively), while the aperture of the groove is defined by the angle $\theta_{v}=\pi-2\left|\theta_{\mathrm{s}}\right|$
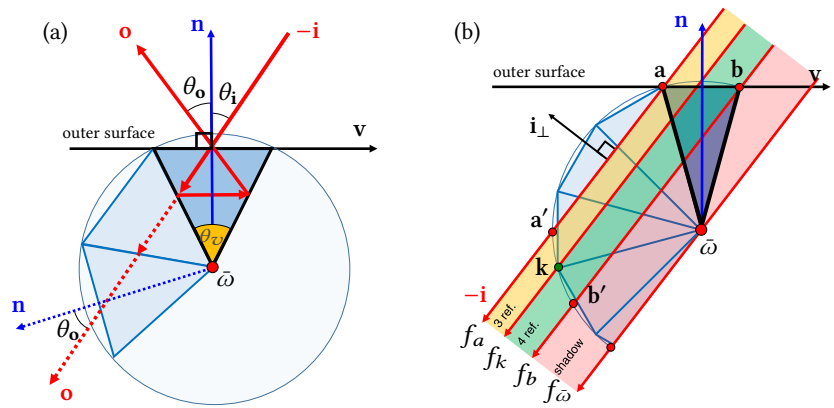

Fig. 4. Kaleidoscopic analysis for symmetric V-grooves. (a) To simulate specular light transport inside the groove, we create virtual mirror grooves around the reflecting facet, allowing the incident light to be collinear. Our kaleidoscopic reflection model allows formulating multiple reflections in a groove as a line equation in the n-s plane. (b) An example of three and four bounces in a symmetric $\mathrm{V}$-groove. Depending on the angle and position of the incident light, we can determine the number of reflections $k$, as well as the geometric term $G(\mathbf{i}, \mathbf{o}, \mathbf{s})$.

for symmetric grooves, and how multiple reflections are handled with it. Then, we extend this analysis to nonsymmetric V-grooves.

\subsection{Multiple Reflections in a Symmetric V-Groove}

Kaleidoscopic V-groove model. Figure 3 depicts the classic view of microfacet $\mathrm{V}$-grooves, extending to infinite in the cavity axis $\mathbf{u}$, and with facet orientation $\mathbf{s}=\left(\phi_{\mathbf{v}}, \theta_{\mathbf{s}}\right)$ perpendicular to $\mathbf{u}$. Vectors $\mathbf{n}, \mathbf{i}=\left(\phi_{\mathbf{i}}, \theta_{\mathbf{i}}\right)$, and $\mathbf{o}=\left(\phi_{\mathbf{o}}, \theta_{\mathbf{o}}\right)$ represent the surface normal, and the incident and exitant rays, respectively. In our coordinate system, a clockwise angle is negative while counterclockwise is positive. Figure $3 \mathrm{~b}$ shows the same geometry looking down the $\mathbf{u}$ direction (perpendicular to the $\mathbf{n}$-s plane, where $\mathbf{s}$ is the facet normal).

Figure 4a illustrates in 2D how our kaleidoscopic model is built. Instead of calculating intersections and mirror reflections within a groove until the ray finally abandons it (red solid lines traversing the dark blue triangle), we create a straight line in the incoming direction $\mathbf{i}$ (red dotted line) and create a virtual groove (light blue triangle) which is a mirror reflection of the previous one, with a shared edge (the mirror-reflection version of a symmetric groove looks identical; however, the shape difference will become obvious 
when introducing nonsymmetric grooves). This "unfolding" of virtual grooves continues until the line leaves their bounding circle (of unit length). This results in a simple geometry which, as we will see next, allows us to compute multiple scattering in a fast and analytical way.

Number of internal reflections. Our kaleidoscopic model allows us to bypass costly computations for multiple scattering within a groove; instead, we simply need to compute a few geometric variables. The first interesting property of our model is the fact that the number of reflections inside the real groove is determined by the number of intersections of the straight line with the edges of the grooves. Moreover, an incident ray can only go through $k-1$ or $k$ reflections, depending on the incident angle $\theta_{\mathbf{i}}$, the incident point, and the $\mathrm{V}$-groove angle (please refer to Appendix B for proof). Given that $\theta_{v}=\pi-2\left|\theta_{\mathbf{s}}\right|$, this $k$ number is given by

$$
k=\left\lfloor\left(\pi+2 \theta_{\mathbf{i}}\right) /\left(\pi-2\left|\theta_{\mathbf{s}}\right|\right)\right\rfloor+1 .
$$

We now need to define the transition point $\mathbf{k}$ between $k-1$ or $k$ reflections. As Figure $4 \mathrm{~b}$ shows, we define such point $\mathrm{k}$ on the bounding circle surrounding the grooves in our kaleidoscopic model. Intuitively, if we mirror points $\mathbf{a}$ and $\mathbf{b}$ with respect to $\mathbf{i}_{\perp}$ (the axis perpendicular to the incident direction passing through the center of the circle), we obtain points $\mathbf{a}^{\prime}$ and $\mathbf{b}^{\prime}$, also on the bounding circle. Point $\mathbf{k}$ must lie between $\mathbf{a}^{\prime}$ and $\mathbf{b}^{\prime}$, since $\theta_{k}=k \theta_{v}-\theta_{v} / 2$. A more detailed explanation is also offered in Appendix B.

Outgoing direction. The outgoing direction o of the ray leaving the groove after multiple bounces can be computed as a function of the incident direction $\mathbf{i}$ and the facet orientation $\mathbf{s}$, where

$\theta_{\mathbf{o}}= \begin{cases}(-1)^{k-1}\left(\theta_{\mathbf{i}}+\pi-(k-1)\left(\pi-2\left|\theta_{\mathbf{s}}\right|\right)\right), & (k-1) \text { reflections, } \\ (-1)^{k}\left(\theta_{\mathbf{i}}+\pi-k\left(\pi-2\left|\theta_{\mathbf{s}}\right|\right)\right), & (k) \text { reflections. }\end{cases}$

Geometric attenuation term. To come up with a geometric attenuation term $G(\mathbf{i}, \mathbf{o}, \mathbf{s})$ analogous to the shadowing-masking term in the Cook-Torrance model, we define line equations parallel to the incident direction $\mathbf{i}$ as $f(\mathbf{p})=\mathbf{i}_{\perp} \cdot \mathbf{p}$, where $\mathbf{p}$ represents an arbitrary point. The output value of function $f$ indicates the orthogonal offset of the line away from the origin $\bar{\omega}$. We can thus define a novel geometric attenuation factor $G(\mathbf{i}, \mathbf{o}, \mathbf{s})$ from simple relations as

$$
G(\mathbf{i}, \mathbf{o}, \mathbf{s})= \begin{cases}\left(f_{a}-f_{k}\right) /\left(f_{a}\right), & (k-1) \text { reflections } \\ \left(f_{k}-f_{b}\right) /\left(f_{a}\right), & (k) \text { reflections }\end{cases}
$$

where $f_{a}, f_{b}$, and $f_{k}$ represent offset distances from $\bar{\omega}$, obtained from $f(\mathbf{a}), f(\mathbf{b})$, and $f(\mathbf{k})$. Note that if $f_{b}<0$ then light is arriving to the opposite facet; since this case should be handled by mirroring over that facet, we set $f_{b}=\max (f(\mathbf{b}), 0)$.

Figure $4 \mathrm{~b}$ shows an example for the case of $k=4$. Rays within the $f_{a}-f_{k}$ zone (yellow) will be reflected three times, while rays in the $f_{k}-f_{b}$ zone (green) will be reflected four times. The $f_{b}-f_{\bar{\omega}}$ zone represents the shadow area.

\subsection{Multiple Reflections in a Nonsymmetric V-Groove}

Now we describe how to extend our kaleidoscopic model to nonsymmetric grooves, and explain how to handle multiple reflections inside them.

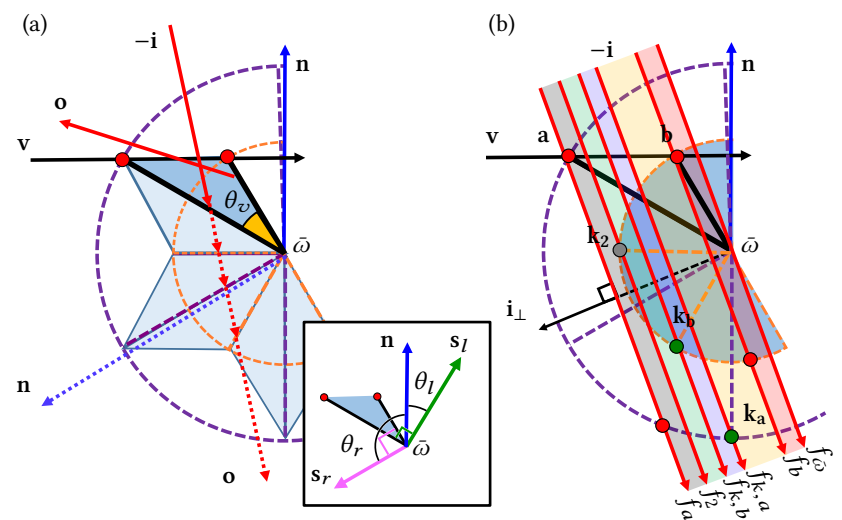

Fig. 5. Kaleidoscopic analysis for nonsymmetric V-grooves, defined by the left and right angles $\theta_{l}$ and $\theta_{r}$ respectively, where the aperture of the groove is $\theta_{v}=\left|\left(\theta_{l}+\pi / 2\right)-\left(\theta_{r}-\pi / 2\right)\right|=\left|\pi+\theta_{l}-\theta_{r}\right|$. (a) As in the symmetric case, we build our kaleidoscopic model by mirroring the groove over its reflecting facet, creating a fan of non-isosceles triangles. By joining the inner and outer vertices we create two concentric circles bounding each facet (purple and orange dotted circles). As in the symmetric case, this geometry allows to model multiple specular reflections in the groove as a line equation, since the incident vector $\mathbf{i}$ remains collinear. The inset shows the facet angles $\theta_{l}$ and $\theta_{r}$. (b) An example of the geometric attenuation showing single scattering, two, three, and four bounces inside the V-groove, and shadowing.

Kaleidoscopic nonsymmetric V-groove model. Let us define again our reference system with respect to the normal $\mathbf{n}$. In the nonsymmetric case, we have two facet orientations for the left and right facets, $\mathbf{s}_{l}$ and $\mathbf{s}_{r}$; the angle between the normal $\mathbf{n}$ and each facet is given by $\theta_{l}$ and $\theta_{r}$. As in the symmetric case, we iteratively mirror the $\mathrm{V}$-groove counter-clockwise along the left edge, creating a fan of non-isosceles triangles. As a result, we no longer have one single bounding circle connecting all triangles' outer vertices, but two, as shown in Figure 5a. As a consequence, in order to compute the number of intersections of a ray within the groove analytically, we now need to evaluate the intersections with both circles. Effectively, this is equivalent to considering two overlapping symmetric groove fans with aperture $2 \theta_{v}$, each of them starting on each physical facet of the groove (purple and orange dashed wedges in Figure 5a).

Number of internal reflections. Analogous to the symmetric Vgroove model, we can compute the number of reflections of a ray as a function of its incident direction $\mathbf{i}$ and incident position. However, we now need to compute this for the two overlapping sets of triangles, leading to two $k$ numbers, $k_{\mathrm{a}}$ and $k_{\mathrm{b}}$, where

$$
k_{\mathbf{b}}=2\left\lfloor\left(\pi+2 \theta_{\mathbf{i}}-\left(\theta_{l}+\theta_{r}\right)\right) /\left(2 \theta_{v}\right)+0.5\right\rfloor,
$$

and $k_{\mathrm{a}}=k_{\mathrm{b}}+1$.

Outgoing direction. We compute the outgoing direction o of the ray leaving the nonsymmetric groove after $k$ bounces as a function of the incident direction $\mathbf{i}$ as

$$
\theta_{\mathbf{o}}=(-1)^{k}\left(\theta_{\mathbf{i}}-\frac{\theta_{l}+\theta_{r}}{2}+\pi-k\left(\pi+\theta_{l}-\theta_{r}\right)\right)+\frac{\theta_{l}+\theta_{r}}{2} .
$$

Geometric attenuation term. Given the groove points $\mathbf{a}$ and $\mathbf{b}$, and the transition points $\mathbf{k}_{\mathbf{a}}$ and $\mathbf{k}_{\mathbf{b}}$, we can define $f_{a}, f_{b}, f_{k, a}$, and 
$f_{k, b}$ using $\max (0, f(\mathbf{p}))$. In addition, in order to check whether light enters the groove or is directly reflected (single scattering), we need to define $f_{2}=f\left(\mathbf{k}_{2}\right)$, with $\mathbf{k}_{2}$, the equivalent of point $\mathbf{b}$ in the adjacent mirrored groove, so that $\theta_{2}=\theta_{r}-\pi / 2+2 \theta_{v}$. These five equations create five different ray zones as shown in Figure $5 \mathrm{~b}$, taking into account single scattering, $k_{b}-1, k_{b}, k_{b}+1$ reflections, and shadowing, respectively. We therefore define our geometric attenuation term as

$$
G\left(\mathbf{i}, \mathbf{o}, \mathbf{s}_{l}, \mathbf{s}_{r}\right)= \begin{cases}\max \left(0, f_{a}-\max \left(f_{2}, f_{b}\right)\right) / f_{a}, & 1 \text { refl. } \\ \max \left(0, \min \left(f_{a}, f_{2}\right)-f_{k, b}\right) / f_{a}, & k_{b}-1 \text { refl. } \\ \max \left(0, \min \left(f_{a}, f_{k, b}\right)-\max \left(f_{k, a}, f_{b}\right)\right) / f_{a}, & k_{b} \text { refl. } \\ \max \left(0, \min \left(f_{k, b}, f_{k, a}\right)-f_{b}\right) / f_{a}, & k_{b}+1 \text { refl. }\end{cases}
$$

Note that we have assumed that the incident ray hits the left surface first; the model however supports intersecting at the right surface simply by setting $\theta_{\mathbf{i}}=-\theta_{\mathbf{i}}, \theta_{l}=-\theta_{r}$ and $\theta_{r}=-\theta_{l}$.

\section{A MULTIPLE-SCATTERING-AWARE BRDF}

Based on the analysis performed in Section 4, we now develop a novel, closed-form BRDF model that extends the traditional microfacet theory [Torrance and Sparrow 1967], supporting multiple scattering even for nonsymmetric $\mathrm{V}$-grooves. We share the following assumptions with the original model:

- Each facet corresponds to one side of a V-groove cavity.

- The longitudinal axis of the V-groove cavity is parallel to the object surface (i.e., perpendicular to the macroscopic normal)

- All the peak points formed by two adjacent grooves lie on the same object plane; as a result shadowing, masking, and interreflections only occur within a single cavity, and not between multiple cavities.

- Light transport within a V-groove is perfectly specular.

From this common ground, our extended model differs from the original microfacet theory in two key points:

- Light can scatter multiple times before leaving the V-groove.

- The shape of the V-groove is not necessarily symmetric with respect to the object surface normal.

For simplicity, we first present the case of symmetric V-grooves, then generalize it to support asymmetry.

\subsection{Symmetric V-groove BRDF}

Let us define the differential flux $d \Phi_{\mathbf{i}, \mathbf{s}}$ incident from direction $\mathbf{i}$ into a set of symmetric V-grooves with normal $\mathbf{s}$ as

$$
\mathrm{d} \Phi_{\mathbf{i}, \mathbf{s}}=L_{i}(\mathbf{i}) \operatorname{did} A^{\perp}(\mathbf{s})=L_{i}(\mathbf{i}) \mathrm{d} \mathbf{i}|\mathbf{i} \cdot \mathbf{s}| D(\mathbf{s}) \mathrm{d} \mathbf{s} \mathrm{d} A,
$$

where $L_{i}(\mathbf{i})$ di is the differential irradiance from direction $\mathbf{i}$, di is the solid angle, $\mathrm{d} A^{\perp}(\mathbf{s})$ is the projected differential area of the facets in direction $\mathbf{i}$, and $D(\mathbf{s})$ the distribution of normals $\mathbf{s}$.

As light scatters multiple times within the V-groove, it loses energy. Since we assume that the surfaces are perfect mirrors, this energy loss is modeled using the Fresnel reflection equation. Based on the topological analysis of our kaleidoscopic model in Section 4 , we can compute the differential outgoing flux $d \Phi_{\mathbf{o}, \mathbf{s}}$ in the direction o after $k$ bounces as

$$
\mathrm{d} \Phi_{\mathbf{o}, \mathbf{s}}=\prod_{j=1}^{k} F\left(\mathbf{i} \cdot \mathbf{s}^{j}\right) \mathrm{d} \Phi_{\mathbf{i}, \mathbf{s}},
$$

where $F$ is the standard Fresnel reflection equation, and $\mathbf{s}^{j}$ is the facet normal at the $j$-th bounce.

We can express the differential outgoing radiance $\mathrm{d} L_{o}(\mathbf{o})$ reflected by a given facet in terms of the geometric attenuation term $G(\mathbf{i}, \mathbf{o}, \mathbf{s})$ [Equation (6)] as follows:

$$
\begin{aligned}
\mathrm{d} L_{\boldsymbol{o}}(\mathbf{o}) & =\frac{\mathrm{d} \Phi_{\mathbf{o}, \mathbf{s}}}{\mathrm{d} A^{\perp} \mathrm{d} \mathbf{o}} \\
& =\frac{\mathrm{d} \mathbf{s}}{\mathrm{d} \mathbf{o}} \frac{\prod_{j=1}^{k} F\left(\mathbf{i} \cdot \mathbf{s}^{j}\right)|\mathbf{i} \cdot \mathbf{s}| D(\mathbf{s}) G(\mathbf{i}, \mathbf{o}, \mathbf{s})}{|\mathbf{i} \cdot \mathbf{n}||\mathbf{o} \cdot \mathbf{n}|} L_{i}(\mathbf{i})|\mathbf{i} \cdot \mathbf{n}| \mathrm{d} \mathbf{i},
\end{aligned}
$$

Finally, given the definition of the $\operatorname{BRDF} \rho(\mathbf{i}, \mathbf{o})=\frac{\mathrm{d} L_{o}(\mathbf{o})}{L_{i}(\mathbf{i})|\mathbf{i} \cdot \mathbf{n}| \mathrm{di}}$, and the projection factor $\frac{\mathrm{d} s}{\mathrm{~d}}$ (see Appendix A)

$$
\left|\frac{\mathrm{d} \mathbf{s}}{\mathrm{d} \mathbf{o}}\right|=\frac{\sin \theta_{\mathbf{s}}}{k \sin \theta_{\mathbf{h}}} \frac{1}{4 \cos \theta_{\mathbf{d}}}
$$

where $\theta_{\mathbf{h}}$ is the angle between $\mathbf{h}$ and $\mathbf{n}$, and $\theta_{\mathbf{d}}$ is the angle between $\mathbf{h}$ and $\mathbf{i}$, following Rusinkiewicz's parameterization [1998], we can combine individual reflections and define a BRDF for each microfacet as a function of $k$, as follows:

$$
\rho(\mathbf{i}, \mathbf{o} ; \mathbf{s}, k)=\frac{\sin \theta_{\mathbf{s}}}{k \sin \theta_{\mathbf{h}}} \frac{\prod_{j=1}^{k} F\left(\mathbf{i} \cdot \mathbf{s}^{j}\right)|\mathbf{i} \cdot \mathbf{s}| D(\mathbf{s}) G(\mathbf{i}, \mathbf{o}, \mathbf{s})}{4 \cos \theta_{\mathbf{d}}|\mathbf{i} \cdot \mathbf{n}||\mathbf{o} \cdot \mathbf{n}|} .
$$

Equation (5) established the relationship between $\theta_{\mathbf{o}}, \theta_{\mathbf{i}}, \theta_{\mathbf{s}}$, and the number of reflections $k$. From that equation, it can be shown that computing $\theta_{\mathbf{s}}$ from given values of $\theta_{\mathbf{o}}, \theta_{\mathbf{i}}$, and $k$ leads to two possible solutions. The physical interpretation is that, in the presence of multiple scattering inside the V-groove, two different microfacet orientations $\mathbf{s}_{1}$ and $\mathbf{s}_{2}$ can end up reflecting light in the same direction o, given an incident direction $\mathbf{i}$. This in turn means that to compute the total differential outgoing flux $\mathrm{d} \Phi_{\mathbf{o}}$ we need to take into account the contributions from both orientations [each given by Equation (11)] as

$$
\mathrm{d} \Phi_{\mathbf{o}}=\mathrm{d} \Phi_{\mathbf{o}, \mathbf{s}_{1}}+\mathrm{d} \Phi_{\mathbf{o}, \mathbf{s}_{2}} .
$$

By plugging Equation (15) into Equation (12), we derive our $k$ resolved BRDF taking into account multiple scattering:

$$
\rho(\mathbf{i}, \mathbf{o} ; k)=\sum_{m=1}^{2} \frac{\sin \theta_{\mathbf{s}_{m}}}{k \sin \theta_{\mathbf{h}}} \frac{\prod_{j=1}^{k} F\left(\mathbf{i} \cdot \mathbf{s}_{m}^{j}\right)|\mathbf{i} \cdot \mathbf{s}| D\left(\mathbf{s}_{m}\right) G\left(\mathbf{i}, \mathbf{o}, \mathbf{s}_{m}\right)}{4 \cos \theta_{\mathbf{d}}|\mathbf{i} \cdot \mathbf{n}||\mathbf{o} \cdot \mathbf{n}|}
$$

where $m$ refers to the two different facet orientations $\mathbf{s}_{1}$ and $\mathbf{s}_{2}$. Finally, we get the complete BRDF as

$$
\rho(\mathbf{i}, \mathbf{o})=\sum_{k=1}^{N} \rho(\mathbf{i}, \mathbf{o} ; k),
$$

where $N$ is the maximum number of bounces within a $\mathrm{V}$-groove cavity. Note that $N$ can be potentially infinite; however, we have observed that even for high roughness $N$ remains relatively low. We discuss this in more depth in Section 8. 


\subsection{Nonsymmetric V-groove BRDF}

Defining a nonsymmetric V-groove distribution. Following our analysis in Section 4, we now define a nonsymmetric V-groove by the two orientation vectors of its facets, $\mathbf{s}_{l}$ and $\mathbf{s}_{r}$ (Figure 5a). For our stochastic nonsymmetric BRDF we first need to define a bivariate distribution of normals $D\left(\mathbf{s}_{l}, \mathbf{s}_{r}\right)=P\left(\mathbf{s}_{r} \mid \mathbf{s}_{l}\right) D\left(\mathbf{s}_{l}\right)$, where $P\left(\mathbf{s}_{r} \mid \mathbf{s}_{l}\right)$ is the conditional probability distribution of $\mathbf{s}_{r}$ given $\mathbf{s}_{l}$, and $D\left(\mathbf{s}_{l}\right)$ is the NDF of the microsurface (e.g., Beckmann or GGX).

The distributions of left and right facets in a V-groove can be defined using a univariate distribution function $D$, leading to $D\left(\mathbf{s}_{r}\right)$ and $D\left(\mathbf{s}_{l}\right)$, respectively. The stochastic relationship between $D\left(\mathbf{s}_{r}\right)$ and $D\left(\mathbf{s}_{l}\right)$ can be calculated from the projected areas of $\mathbf{s}_{r}$ and $\mathbf{s}_{l}$. The differential projected area of the right facets $\mathrm{d} A\left(\mathbf{s}_{r}\right)$ is obtained by $D\left(\mathbf{s}_{r}\right) \cos \theta_{r} \mathrm{~d} \mathbf{s}_{r}$, and similarly for the left facets as $\mathrm{d} A\left(\mathbf{s}_{l}\right)=$ $D\left(\mathbf{s}_{l}\right) \cos \theta_{l} \mathrm{~d} \mathbf{s}_{l}$. We can formulate $\mathrm{d} A\left(\mathbf{s}_{r}\right)$ as the integral of $\mathrm{d} A\left(\mathbf{s}_{l}\right)$ over the solid angle $\Omega$, with a geometric factor $A_{r}$ over $A_{l}$ :

$$
\mathrm{d} A\left(\mathbf{s}_{r}\right)=\int_{\Omega} \frac{A_{r}}{A_{l}} \mathrm{~d} A\left(\mathbf{s}_{l}\right) .
$$

Both $\mathbf{s}_{l}$ and $\mathbf{s}_{r}$ lie on the plane perpendicular to the $\mathbf{u}$-axis; we can thus define the V-groove with three variables $\phi_{\mathbf{v}}, \theta_{l}$, and $\theta_{r}$ (as shown in Figure 5). We then can reparameterize $P\left(\mathbf{s}_{r} \mid \mathbf{s}_{l}\right)$ as $P\left(\theta_{r} \mid \theta_{l}\right)$. To compute $P\left(\theta_{r} \mid \theta_{l}\right)$, we leverage the relationship between the two facets, whose projected areas follow the ratio of $\frac{\tan \theta_{l}}{\tan \theta_{r}}$. The projected area of the right facet $A_{r}$ can then be expressed as a function of the projected area of the left facet $A_{l}$, as $A_{r}=$ $P\left(\theta_{r} \mid \theta_{l}\right) \mathrm{d} \theta_{r} \frac{\tan \theta_{l}}{\tan \theta_{r}} A_{l}$. By substituting $\mathrm{d} A\left(\mathbf{s}_{r}\right), \mathrm{d} A\left(\mathbf{s}_{l}\right)$, and $\frac{A_{r}}{A_{l}}$ in Eq. (18) with $D\left(\mathbf{s}_{r}\right) \cos \theta_{r} \mathrm{~d} \mathbf{s}_{r}, D\left(\mathbf{s}_{l}\right) \cos \theta_{l} \mathrm{~d} \mathbf{s}_{l}$, and $P\left(\theta_{r} \mid \theta_{l}\right) \mathrm{d} \theta_{r} \frac{\tan \theta_{l}}{\tan \theta_{r}}$ the differential projected area of the right facets $\mathrm{d} A\left(\mathbf{s}_{r}\right)$ can then be defined as follows:

$$
D\left(\mathbf{s}_{r}\right) \cos \theta_{r} \mathrm{~d} \mathbf{s}_{r}=\int_{0}^{\pi / 2}\left(P\left(\theta_{r} \mid \theta_{l}\right) \mathrm{d} \theta_{r} \frac{\tan \theta_{l}}{\tan \theta_{r}} D\left(\mathbf{s}_{l}\right) \cos \theta_{l} \sin \theta_{l} \mathrm{~d} \phi_{\mathbf{v}}\right) \mathrm{d} \theta_{l} .
$$

Finally, the simplest case of $P\left(\theta_{r} \mid \theta_{l}\right)$ models the symmetric orientation of both facets $P\left(\theta_{r} \mid \theta_{l}\right)=\delta\left(\left(-\theta_{r}\right)-\theta_{l}\right)$ : this results in a Dirac delta function, converging to the classic symmetric $\mathrm{V}$-groove. We are however interested in keeping the additional degrees of freedom of nonsymmetric grooves; By assuming that $\theta_{r}$ and $\theta_{l}$ are mutually independent, then $P\left(\theta_{r} \mid \theta_{l}\right)=P\left(\theta_{r}\right)$, which allows us to compute $P\left(\theta_{r}\right)$ as

$$
P\left(\theta_{r}\right)=\frac{D\left(\mathbf{s}_{r}\right) \sin ^{2} \theta_{r}}{\int_{0}^{\frac{\pi}{2}} D\left(\mathbf{s}_{l}\right) \sin ^{2} \theta_{l} \mathrm{~d} \theta_{l}} .
$$

Appendix $\mathrm{C}$ includes the closed form of $P\left(\theta_{r}\right)$ for the Beckmann, Phong, and GGX normal distribution functions. Finally, note that we could introduce the dependency of $\phi_{\mathbf{v}}$ on the distribution function as $P\left(\theta_{r} \mid \phi_{\mathbf{v}}\right)$ without modifying our derivation, thus allowing for anisotropy on the skewness of the grooves.

BRDF for nonsymmetric $V$-grooves. Given the probability distributions $D(\mathbf{s})$ and $P\left(\theta_{r} \mid \theta_{l}\right)$, we can now compute the differential flux $\mathrm{d} \Phi_{\mathbf{s}_{l}}$ incident on the left side of V-groove from direction $\mathbf{i}$ as

$$
\mathrm{d} \Phi_{\mathbf{s}_{l}, \theta_{r}}=L_{i}(\mathbf{i}) \mathrm{d} \mathbf{i}\left|\mathbf{i} \cdot \mathbf{s}_{l}\right| D\left(\mathbf{s}_{l}\right) \mathrm{d} \mathbf{s}_{l} P\left(\theta_{r} \mid \theta_{l}\right) \mathrm{d} \theta_{r} \mathrm{~d} A .
$$

Following similar derivations as the symmetric case, for a given incoming vector $\mathbf{i}$ we need to obtain the V-groove that reflects light in the outgoing direction o after $k$ bounces. Equation (8), however, has two unknowns $\left(\theta_{l}\right.$ and $\left.\theta_{r}\right)$, which means that more than a single groove complies with the reflection condition given by the set of variables $(\mathbf{i}, \mathbf{o}, k)$.

Inverting Equation (8) thus leads to a set of grooves defined by the function $\theta_{l}\left(\theta_{r} ; \mathbf{i}, \mathbf{o}, k\right)$, with $\theta_{r} \in\left[-\frac{\pi}{2}, \frac{\pi}{2}\right]$. The outgoing flux $\mathrm{d} \Phi_{\mathbf{o}}$ in direction $\mathbf{o}$ is the result of all contributing grooves defined by $\theta_{r}$ and the function $\theta_{l}\left(\theta_{r} ; \mathbf{i}, \mathbf{o}, k\right)$, as

$$
\mathrm{d} \Phi_{\mathbf{o}}=\int_{-\pi / 2}^{\pi / 2} \mathrm{~d} \Phi_{\mathbf{o}, \mathbf{s}_{l}, \theta_{l}\left(\theta_{r}\right)},
$$

where $\mathrm{d} \Phi_{\mathbf{o}}, \mathbf{s}_{l}, \theta_{l}\left(\theta_{r}\right)$ is the differential outgoing flux from the groove defined by $\mathbf{s}_{l}=\left(\theta_{l}\left(\theta_{r}\right), \phi_{\mathbf{v}}\right)$ and $\mathbf{s}_{r}=\left(\theta_{r}, \phi_{\mathbf{v}}\right)$ (we remove the rest of parameters for clarity).

Given $\mathrm{d} L_{\boldsymbol{o}}(\mathbf{o})=\frac{\mathrm{d} \Phi_{\mathbf{o}}}{\mathrm{d} A^{\perp} \mathrm{do}}$ and Equation (22), and following the same derivations as in the symmetric case, the $k$-reflection BRDF for nonsymmetric V-grooves can be expressed as

$$
\begin{gathered}
\rho(\mathbf{i}, \mathbf{o} ; k)=\int_{-\pi / 2}^{\pi / 2} \rho\left(\mathbf{i}, \mathbf{o} ; k, \mathbf{s}_{l}, \mathbf{s}_{r}\right) \mathrm{d} \theta_{r}, \\
\rho\left(\mathbf{i}, \mathbf{o} ; k, \mathbf{s}_{l}, \mathbf{s}_{r}\right)=\frac{R\left(\mathbf{i}, \mathbf{o} ; k, \mathbf{s}_{l}, \mathbf{s}_{r}\right)\left|\mathbf{i} \cdot \mathbf{s}_{l}\right| D\left(\mathbf{s}_{l}\right) P\left(\theta_{r} \mid \theta_{l}\right)}{|\mathbf{i} \cdot \mathbf{n}||\mathbf{o} \cdot \mathbf{n}|}\left|\frac{\mathrm{d} \mathbf{s}_{l}}{\mathrm{~d} \mathbf{o}}\right|, \\
\text { where } R\left(\mathbf{i}, \mathbf{o} ; k, \mathbf{s}_{l}, \mathbf{s}_{r}\right)=\prod_{j=0}^{k-1} F\left(\mathbf{i} \cdot \mathbf{s}_{l}^{j}\right) G\left(\mathbf{i}, \mathbf{o}, \mathbf{s}_{l}, \mathbf{s}_{r}\right), \text { and } G\left(\mathbf{i}, \mathbf{o}, \mathbf{s}_{l}, \mathbf{s}_{r}\right)
\end{gathered}
$$
is the attenuation term for nonsymmetric V-grooves, and $\left|\frac{\mathrm{d} s_{l}}{\mathrm{do}}\right|$ is defined as $\frac{\sin \theta_{\mathbf{s}_{l}}}{0.5\left|0.5-(-1)^{k}(k+0.5)\right| \sin \theta_{\mathbf{h}}} \frac{1}{4 \cos \theta_{\mathbf{d}}}$; both $\mathbf{s}_{l}$ and $\mathbf{s}_{r}$ satisfy that light from the incoming direction $\mathbf{i}$ is reflected towards $\mathbf{o}$ after $k$ bounces. Note that Equation (9) describes $G\left(\mathbf{i}, \mathbf{o}, \mathbf{s}_{l}, \mathbf{s}_{r}\right)$ for the case of $\theta_{l}>0$; for $\theta_{l}<0$ we first invert the parameters, then apply $G\left(\mathbf{i}, \mathbf{o}, \mathbf{s}_{l}, \mathbf{s}_{r}\right)$. As in the symmetric case, we evaluate the BRDF by plugging Equations (23) into Equation (17).

\subsection{Relationship with the Cook-Torrance Model}

We show how our general, multiple-scattering BRDF for nonsymmetric V-grooves converges to the Cook-Torrance model when assuming symmetric cavities and single scattering. Equation (23) refers to symmetric grooves by setting $P\left(\theta_{r} \mid \theta_{l}\right)=\delta\left(\theta_{r}+\theta_{l}\right)$. As shown in Section 4.1, this means that only two $\theta_{r}$ values fulfill Equation (5) while having non-zero probability distribution $P\left(\theta_{r} \mid \theta_{l}\right)$. This allows us to compute the integral over $\theta_{r}$, transforming Equation (23) into

$$
\rho(\mathbf{i}, \mathbf{o} ; k)=\sum_{m=1}^{2} \frac{R\left(\mathbf{i}, \mathbf{o} ; k, \mathbf{s}_{m, l}, \mathbf{s}_{m, r}\right)\left|\mathbf{i} \cdot \mathbf{s}_{m, l}\right| D\left(\mathbf{s}_{m, l}\right)}{|\mathbf{o} \cdot \mathbf{n}||\mathbf{i} \cdot \mathbf{n}|}\left|\frac{\mathrm{d} \mathbf{s}_{m, l}}{\mathrm{~d} \mathbf{o}}\right|,
$$

By plugging Equation (13) to Equation (24), we can get Equation (14). Assuming $k=1$ in Equation (14), where only one solution exists, and noting that $\mathbf{s}=\mathbf{h}$ in such case, we get

$$
\begin{aligned}
\rho(\mathbf{i}, \mathbf{o} ; 1) & =\frac{\sin \theta_{\mathbf{h}}}{1 \sin \theta_{\mathbf{h}}} \frac{F(\mathbf{i} \cdot \mathbf{h})|\mathbf{i} \cdot \mathbf{h}| D(\mathbf{h}) G(\mathbf{i}, \mathbf{o}, \mathbf{h})}{4 \cos \theta_{\mathbf{d}}|\mathbf{o} \cdot \mathbf{n}||\mathbf{i} \cdot \mathbf{n}|} \\
& =\frac{F(\mathbf{i} \cdot \mathbf{h}) D(\mathbf{h}) G(\mathbf{i}, \mathbf{o}, \mathbf{h})}{4|\mathbf{o} \cdot \mathbf{n}||\mathbf{i} \cdot \mathbf{n}|},
\end{aligned}
$$

which is the original Cook-Torrance microfacets model. 

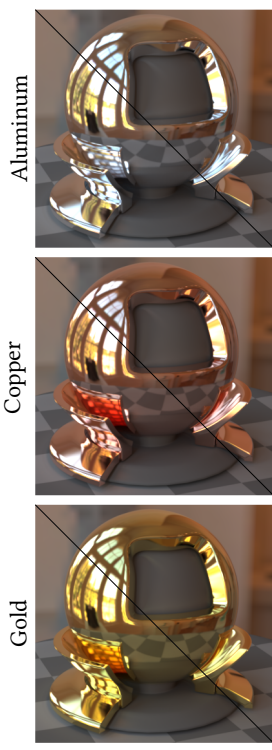

$\alpha=0.01$
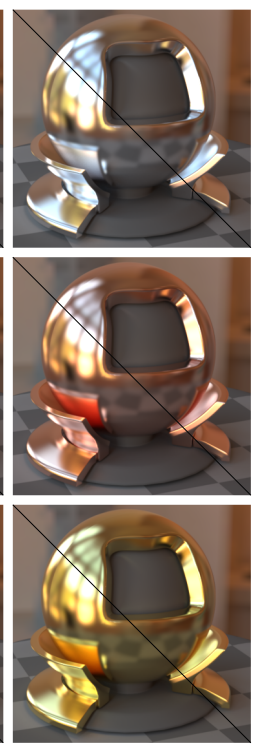

$\alpha=0.05$
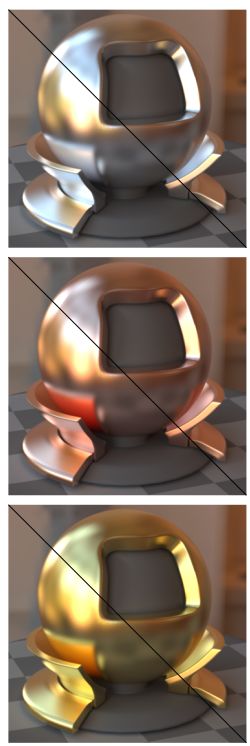

$\alpha=0.1$
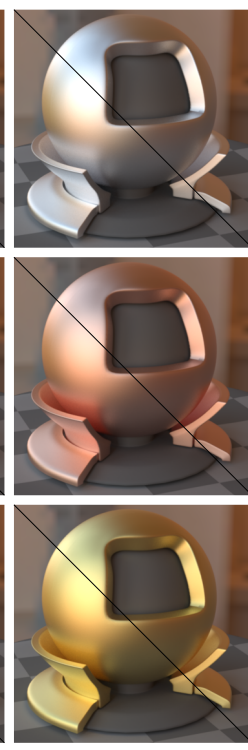

$\alpha=0.3$
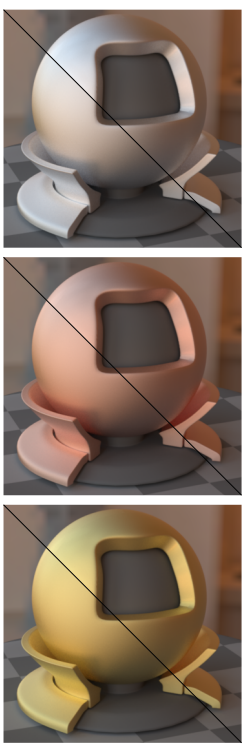

$\alpha=0.5$
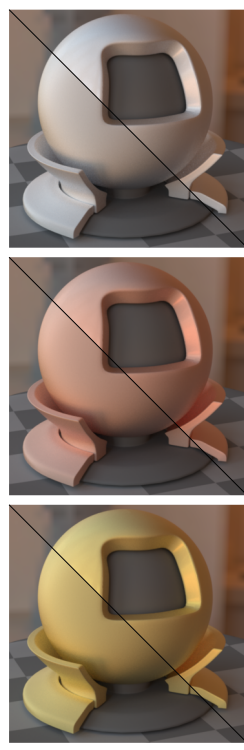

$\alpha=0.7$
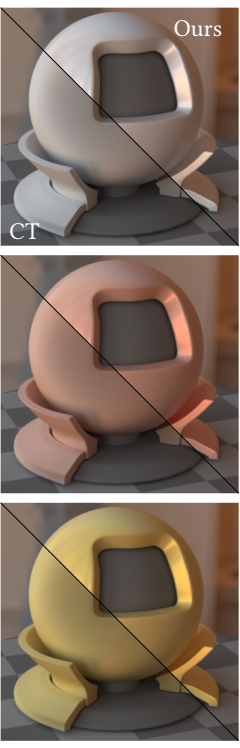

$\alpha=1$

Fig. 6. Comparison between our model including multiple scattering and nonsymmetric V-grooves (top right), against traditional Cook-Torrance model (bottom left), for three different materials. As roughness increases, the effect of multiple scattering becomes more apparent, reintroducing the lost energy due to shadowing-masking and producing more realistic results. Our model only introduces an additional cost of $15 \%$ to $20 \%$ with respect to Cook-Torrance.

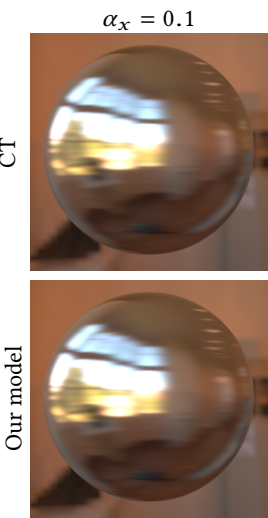

$\alpha_{y}=0.01$
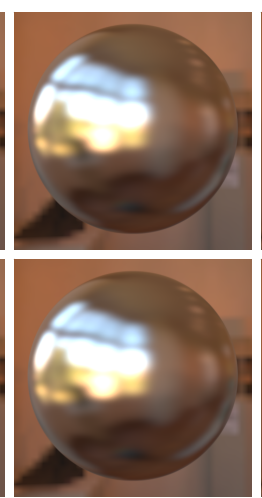

$\alpha_{y}=0.05$

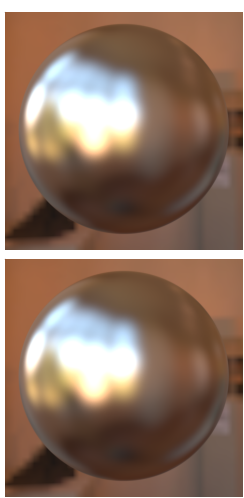

$\alpha_{y}=0.1$
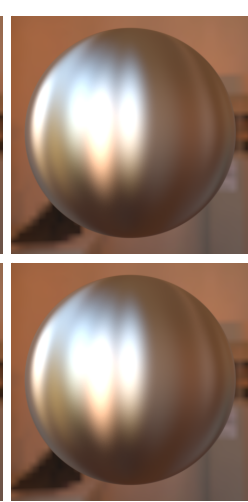

$\alpha_{y}=0.3$

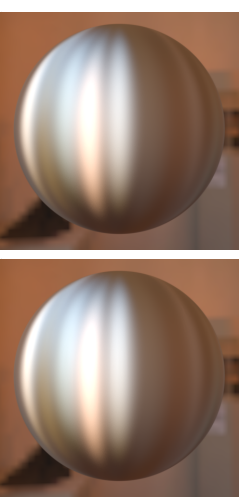

$\alpha_{y}=0.5$

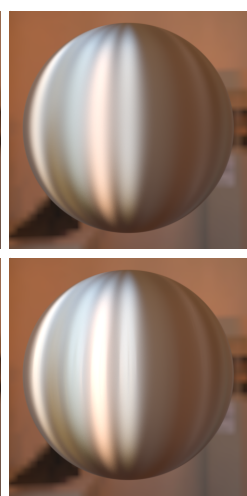

$\alpha_{y}=0.7$

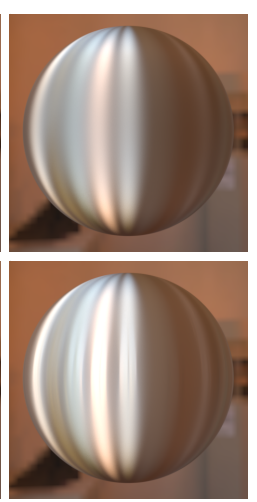

$\alpha_{y}=1$

Fig. 7. Changing the roughness parameter $\alpha_{y}$ in an anisotropic Beckmann NDF ( $\left.\alpha_{x}=0.1\right)$, for an aluminum sphere. As roughness increases, our model preserves its metallic appearance better, especially in highlights.

\section{IMPORTANCE SAMPLING OF THE BRDFS}

As opposed to single scattering BRDFs, the outgoing direction o is not directly defined by the half vector $\mathbf{h}$, since other facets can contribute to a particular direction o via multiple reflections.

Symmetric BRDF. We first sample a vector $\mathbf{s}$, defining the facet hit by the incoming ray in direction $\mathbf{i}$. In particular, we sample $\mathbf{s}$ with a probability density function (PDF): $p(\mathbf{s})=|\mathbf{s} \cdot \mathbf{n}| D(\mathbf{s})$, although other distributions (e.g., the distribution of visible normals [Heitz and d'Eon 2014]) could be used. Then, for a given $\mathbf{s}$ we would like to sample o according to the total radiance reflected towards that direction, which is related to the number of bounces $k$ within the groove. We sample $k$ by computing the geometric term $G$ for all possible bounces (since it is a symmetric V-groove, this means $k$ and $k+1$ bounces), and apply a Russian roulette with a probability $p(k)=G$. Note that $G$ also accounts for masking. Finally, by using Equation (5) we obtain $\mathbf{o}$. The total probability $p(\mathbf{o})$ is therefore $p(\mathbf{o})=|\mathbf{s} \cdot \mathbf{n}| D(\mathbf{s}) G(\mathbf{i}, \mathbf{o}, \mathbf{s})$.

Nonsymmetric BRDF. Sampling nonsymmetric V-grooves requires sampling two different facet normals $\mathbf{s}_{l}$ and $\mathbf{s}_{r}$. Assuming that light hits the left facet first, the former is sampled similarly to symmetric V-grooves, with probability $p\left(\mathbf{s}_{l}\right)=\left|\mathbf{s}_{l} \cdot \mathbf{n}\right| D\left(\mathbf{s}_{l}\right)$; then, we sample $\mathbf{s}_{r}$ using the conditional distribution $P\left(\mathbf{s}_{r} \mid \mathbf{s}_{l}\right)$. Therefore, a V-groove defined by the facets $\mathbf{s}_{l}$ and $\mathbf{s}_{r}$ is sampled with probability distribution $p\left(\mathbf{s}_{l}, \mathbf{s}_{r}\right)=\left|\mathbf{s}_{l} \cdot \mathbf{n}\right| D\left(\mathbf{s}_{l}\right) P\left(\mathbf{s}_{r} \mid \mathbf{s}_{l}\right)$. As in the case of symmetric grooves, we get the outgoing direction $\mathbf{o}$ by sampling the number of bounces $k$ inside the groove applying a Russian roulette on the geometric 
attenuation term $G\left(\mathbf{i}, \mathbf{o}, \mathbf{s}_{l}, \mathbf{s}_{r}\right)$. Finally, we compute $\mathbf{o}$ via Equation (8), with final PDF $p(\mathbf{o})=\left|\mathbf{s}_{l} \cdot \mathbf{n}\right| D\left(\mathbf{s}_{l}\right) P\left(\mathbf{s}_{r} \mid \mathbf{s}_{l}\right) G\left(\mathbf{i}, \mathbf{o}, \mathbf{s}_{l}, \mathbf{s}_{r}\right)$. As discussed in Appendix $\mathrm{C}$, there is no analytical sampling routine for $P\left(\mathbf{s}_{r} \mid \mathbf{s}_{l}\right)$ when derived from the most common normal distribution functions. Therefore, we tabulate its inverse CDF, and sample it in runtime using binary search.

\section{RESULTS AND VALIDATION}

We have implemented our model in Mitsuba [Jakob 2010], as a new rough conductor BSDF. For all our renders, we use a forward path tracer, with the scene illuminated by an environment map. We report render times computed on an Intel Core i7-6700K at $4 \mathrm{GHz}$ with $16 \mathrm{~GB}$ of RAM. Unless otherwise stated, we compare our multiple scattering nonsymmetric BRDF against the original symmetric single scattering Cook-Torrance model, using the Beckmann normal distribution.

Comparison against single scattering. Figure 6 shows a comparison between our model and Cook-Torrance, for increasing roughness $\alpha$ and for three different materials. Cook-Torrance does not account for the increasingly important effect of multiple scattering as roughness increases, resulting in a duller appearance.

Anisotropic materials. Figure 7 shows an anisotropic rough aluminum sphere for varying roughness $\alpha_{y}$ in the $y$-axis, and a fixed moderate roughness in the $x$-axis $\alpha_{x}=0.1$. As roughness increases, the amount of multiple scattering in the microgeometry is higher. Cook-Torrance (top) ignores such interreflections, resulting in an energy loss and less defined highlights.

Symmetric vs. nonsymmetric V-grooves. Figure 8 compares our multiple scattering BRDF for symmetric [Equation (16)] and nonsymmetric V-grooves [Equation (23)]. As the roughness of the surface increases, our nonsymmetric V-groove model scatters energy more evenly, producing more back-scattering in comparison to symmetric V-grooves. Moreover, the additional degree of freedom results into a lower-frequency specular appearance.

Effect of the normal distribution function. Our model inherits the generality of the V-groove microsurface model, and therefore works with any arbitrary distribution of microfacet normals. Figure 9 shows results with three of the most common normal distribution functions: Beckmann, GGX, and Phong.

Textured roughness. Our model supports parameterized spatiallyvarying roughness $\alpha$. Figures 1 and 10 show two scenes scene made up of seven different materials, with roughness values guided by texture maps. Again, Cook-Torrance loses energy in the roughest areas, which results in darker surfaces. Our model, on the other hand, accounts for all light paths within the microsurface.

\subsection{Validation}

We validate the correctness of our model by comparing against brute force numerical simulations, and analyzing energy conservation.

Numerical validation. First, we validate our kaleidoscopic model (Section 4) by analyzing the light transport in a single V-groove in 2D. For each V-groove and direction we generate 1000 random

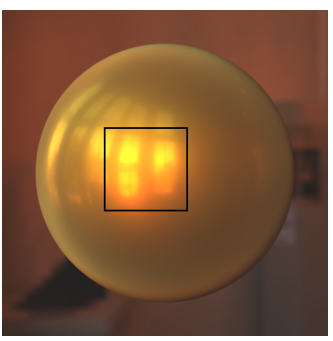

Sym, $\alpha=2.0$

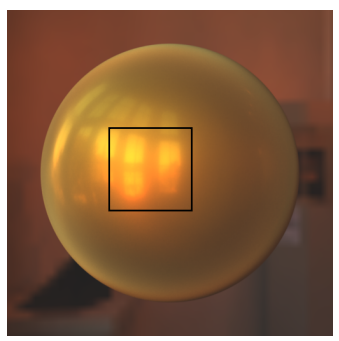

Nonsym, $\alpha=2.0$

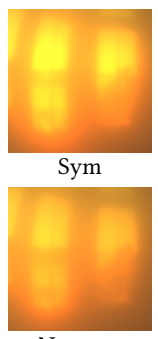

Nonsym
Fig. 8. Comparison between our multiple scattering BRDF for symmetric (left) and nonsymmetric (right) $\mathrm{V}$-grooves. While the nonsymmetric model introduces only subtle differences for low values of roughness $\alpha$, its effect is more noticeable for higher values. In particular, the effect of masking is lower at grazing angles, and the specular reflection due to the singularity at $\mathrm{V}$-grooves is attenuated, as shown in the insets. Note that since our model conserves energy, very high values of roughness $\alpha$ can be applied, which result in significant darkening (lost energy) when using the Cook-Torrance model.

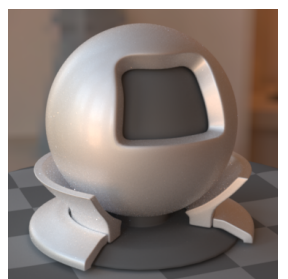

GGX

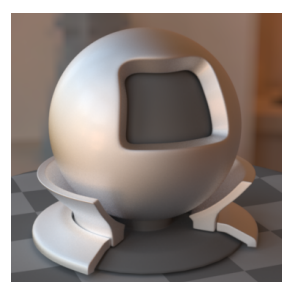

Beckmann

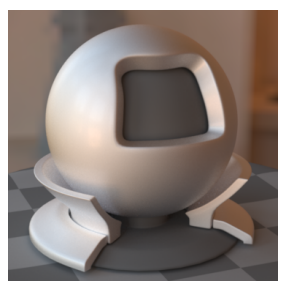

Phong
Fig. 9. Our multiple scattering model does not make any assumption about the underlying NDF modeling the microsurface. The images show rough aluminum spheres with roughness $\alpha=0.5$, using GGX, Beckmann, and Phong NDFs.

Table 1. Error $\epsilon$ (L-1 norm) comparison of our kaleidoscopic model against brute-force Monte Carlo simulations. For the symmetric V-groove, we test 6444 pairs of incident angle $\theta_{\mathbf{i}}$ and $\mathrm{V}$-groove aperture $\theta_{v}$. The incident angle $\theta_{\mathbf{i}}$ ranges from $-89^{\circ}$ to $89^{\circ}$ in steps of $1^{\circ}$, while $\theta_{v}$ ranges from $1^{\circ}$ to $180^{\circ}$ in $5^{\circ}$ steps. For nonsymmetric $\mathrm{V}$-grooves we use the same incident angles $\theta_{\mathbf{i}}$, and vary left and right orientation angles $\theta_{l}$ and $\theta_{r}$ from $5^{\circ}$ to $90^{\circ}$ in steps of $5^{\circ}$, making a total of 324 nonsymmetric $V$-grooves.

\begin{tabular}{|c|c|c|}
\hline model & Symmetric & Nonsymmetric \\
\hline $\max \epsilon$ & 0.003 & 0.004 \\
\hline $\operatorname{avg} \epsilon$ & 0.0005 & 0.0005 \\
\hline
\end{tabular}

walks, and record the number of reflections and outgoing direction of each random walk. We set the Fresnel term to $F=1$ to avoid absorption. We compare the angular response per bounce $k$ with our model, and found an excellent agreement as shown in Table 1.

Figure 11 compares our analytical BRDF for symmetric V-grooves against a brute-force Monte Carlo numerical simulation. For each roughness parameter $\alpha$ and incoming direction $\mathbf{i}$, we average several procedural microsurfaces, generated using the GGX NDF; for each microsurface we sample 10000 random walks within the microsurface. Results with our analytical BRDF closely match the Monte Carlo simulations.

White furnace tests. We validate energy conservation following the same procedure as Heitz et al. [2016]: We render a sphere using 

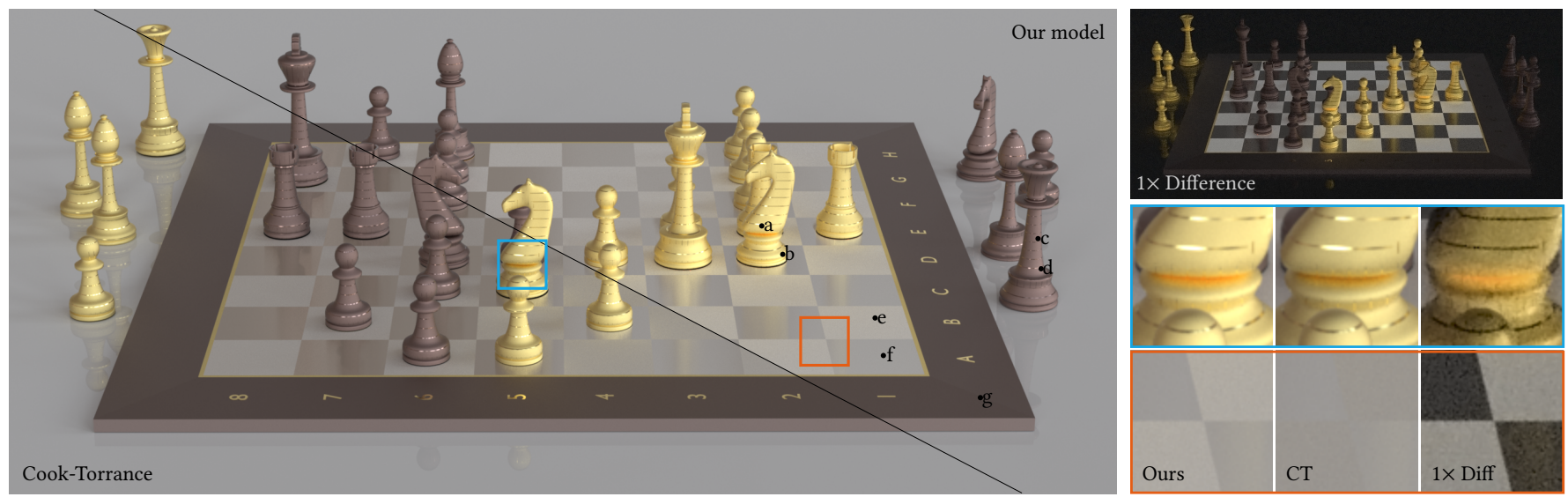

Fig. 10. Chess set made of rough conductors, rendered with Cook-Torrance (CT, bottom-left) and our multiple-scattering-aware microfacet model with support for nonsymmetric V-grooves (top-right). The materials in the scene are: a) Gold, $\alpha=0.05$; b) Gold, $\alpha=0.5$; c) Copper Oxide II, $\alpha=0.5$; d) Copper Oxide II, $\alpha=0.05$; e) Silver, $\alpha=1.0 ;$ f) Silver, $\alpha_{x}=1, \alpha_{y}=0.01$; and g) Copper Oxide I, $\alpha=0.5$. Due to the assumption of single scattering only, Cook-Torrance introduces a significant energy loss (see difference image, top right), especially in the cases of increasingly rough surfaces (see insets). Our model, on the other hand, provides an analytical solution for multiple scattering inside the microsurface. Rendering times for this image with 4096 samples per pixel were CT: 70 min vs Ours: $78 \mathrm{~min}$
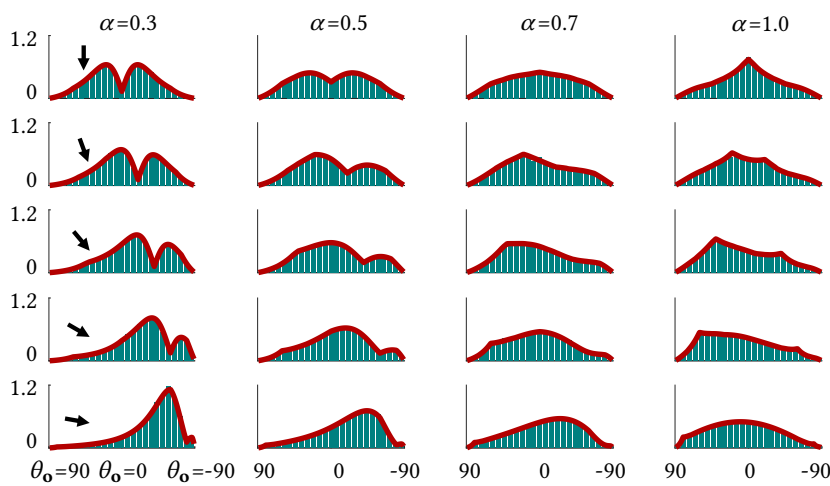

Fig. 11. Validation of our symmetric V-groove BRDF (Equation (16), in red) against a brute-force Monte Carlo simulation of light transport inside the microsurface (green bars), for $\alpha=0.3,0.5,0.7$ and 1 , under varying incident illumination angles. From top to bottom: $\theta_{\mathbf{i}}=0^{\circ}, 20^{\circ}, 40^{\circ}, 60^{\circ}$, and $80^{\circ}$. The microsurface normals are defined using the GGX NDF. Results for other distributions (Beckmann and Phong) can be found in the supplemental material.

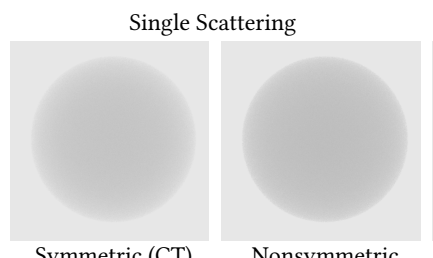

Symmetric (CT)

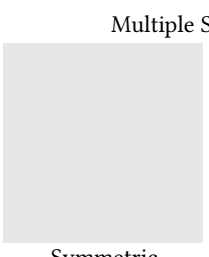

Symmetric

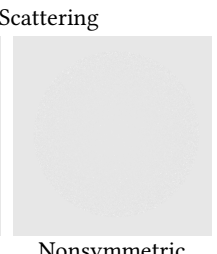

Nonsymmetric
Fig. 12. White furnace tests for our symmetric and nonsymmetric V-groove BRDFs, for single and multiple scattering. Single scattering in symmetric V-grooves corresponds to the Cook-Torrance model. We use the GGX distribution with roughness $\alpha=0.5$, perfect mirror reflection, and Fresnel term $F=1$. Introducing multiple scattering effectively makes the sphere disappear, as expected. our BRDF with Fresnel term $F=1$, and illuminated using a white environment map. Under this scenario, the object should become invisible. Figure 12 shows the results of the test, for single and multiple scattering, and both symmetric and nonsymmetric V-grooves. As expected, the spheres rendered using our multiple scattering BRDF vanish into the white probe.
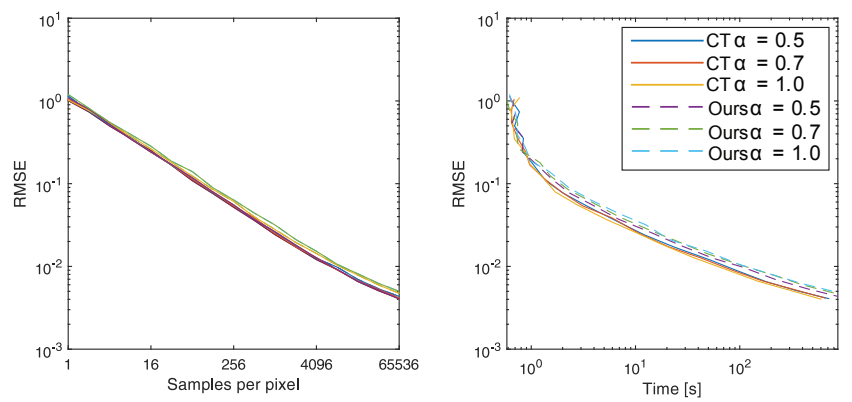

Fig. 13. Convergence comparison between Cook-Torrance and our BRDF, for different roughness $\alpha$, as a function of the number of samples (left) and rendering cost (right). Despite introducing multiple scattering in our model, it does not significantly add cost nor variance.

Convergence. Figure 13 shows the convergence plots for CookTorrance and our method, both as a function of the samples per pixel and time (in seconds). Our model lifts the single-scattering limitation, without significantly increasing rendering costs or variance.

\section{DISCUSSION \& CONCLUSIONS}

We have generalized the $\mathrm{V}$-groove cavity model, presenting an analytical BRDF model that accounts for multiple scattering without the need to compute it explicitly. Our model is based on a novel representation of light transport inside a V-groove, is reciprocal, 


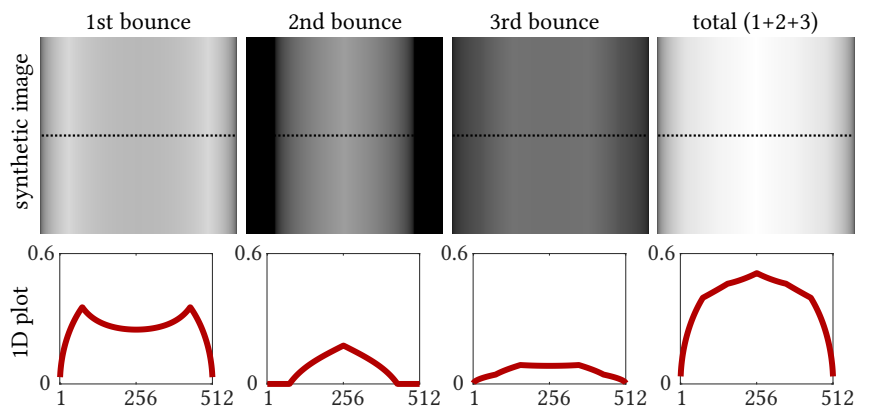

Fig. 14. BRDF discontinuities, inherited from the original V-groove model. We render a cylinder made up of brushed metal using a GGX distribution, and show each scattering order separately (first bounce is the equivalent to the Cook-Torrance model), as well as the final result. The top row shows the rendered images, while the bottom row shows intensity plots along the dotted lines.

conserves energy, has a small computational impact, and has been thoroughly analyzed against Monte Carlo simulations. Our results illustrate the impact of multiple scattering on the final material appearance, specially at high roughness values $\alpha$.

Specular appearance in multiple scattering. Interestingly, as shown in, e.g., Figure 1, multiple scattering inside V-grooves results into a more specular appearance for high roughness values. This departs from the assumption of diffuse-like multiple scattering in the CookTorrance model. This caustic-like behavior emerges in our model due to the mapping between outgoing directions $\mathbf{o}$ and microfacets $\mathbf{s}$, in which multiple facet orientations reflect radiance towards the direction of mirror reflection. Thus, in contrast to single scattering, multiple scattering results in a singularity at mirror reflections. This is mathematically expressed by the $\frac{1}{\sin \theta_{\mathbf{h}}}$ term in Equation (14), which only cancels out when $\sin \theta_{\mathbf{s}} \approx \sin \theta_{\mathbf{h}}$.

Discontinuities. In the original Cook-Torrance model, the geometric attenuation factor for shadowing and masking effects causes discontinuities in the resulting BRDF. We inherit this limitation in our multiple-scattering model, since the differentials for the shadowing and masking cases are different. Given the discrete nature of multiple scattering in V-grooves, such discontinuities are present in each $k$-resolved BRDF $\rho(\mathbf{i}, \mathbf{o} ; k)$, adding up in the final BRDF (see Figures 11 and 14)

Number of bounces. Here we analyze the maximum number of bounces $N$ as a function of roughness and normal distribution NDF From Equation (4), we can see that, as $\theta_{v}$ decreases (i.e., the facet orientation $\mathbf{s}$ gets more perpendicular with respect to $\mathbf{n}$ ) the number of bounces increase. Following this relationship, we analyze the number of bounces for different percentiles of $D(\mathbf{s})$ in Table 2 . Compared to other distributions, the Beckmann distribution only increases slightly the number of maximum bounces; the main conclusion is that the Beckmann distribution leads to less specularity when accounting for multiple scattering, compared to Phong or GGX (see Figure 9).

Comparison with Heitz et al. [2016]. The core difference between the work of Heitz et al. and ours is the microsurface model: While they use the Smith model, we rely on V-grooves. This allows us to

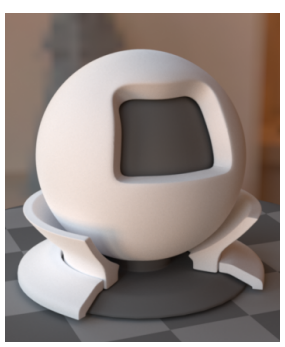

Heitz et al. [2016]

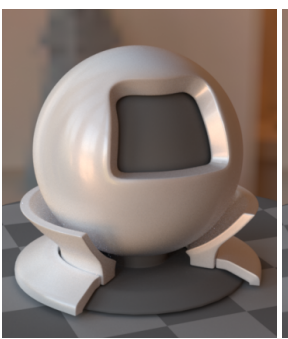

Ours (Nonsymmetric)

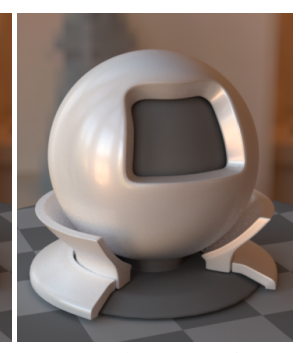

Ours (Symmetric)
Fig. 15. Qualitative comparison between the results of Heitz et al. [2016] and our model for an aluminum geometry with roughness $\alpha=1.0$. Due to the different underlying microgeometry model (Smith vs. V-grooves), the appearance of multiple scattering differs significantly between the stochastic model of Heitz et al., and our analytic closed form model.

Table 2. Maximum bounces in terms of roughness and distribution types for symmetric V-grooves, for different percentiles of the NDF.

\begin{tabular}{|c|ccc|ccc|ccc|}
\hline NDF $D(\mathbf{s})$ & \multicolumn{3}{|c|}{ Beckmann } & \multicolumn{3}{c|}{ Phong } & \multicolumn{3}{c|}{ GGX } \\
\hline Roughness & 0.5 & 0.7 & 1.0 & 0.5 & 0.7 & 1.0 & 0.5 & 0.7 & 1.0 \\
\hline 90\% perc. & 2 & 3 & 3 & 2 & 3 & 5 & 3 & 4 & 5 \\
95\% perc. & 2 & 3 & 3 & 3 & 4 & 7 & 4 & 5 & 7 \\
99\% perc. & 3 & 3 & 4 & 3 & 5 & 16 & 8 & 12 & 16 \\
\hline
\end{tabular}

compute multiple scattering analytically, instead of relying on expensive, numerical stochastic simulations, which in turn makes our model more efficient, and easier to integrate in a render engine. The second main difference is the type of multiple scattering resulting for each microsurface: Because of the uncorrelation assumed by the Smith model, its multiple scattering results into a blurred version of the single scattering lobe. In contrast, the correlation of the Vcavities leads to an increase in frequency in the direction of mirror reflection. There is thus a trade-off between the accuracy of the Smith model and the efficiency of our closed form solution ensuring energy-conservation. We compare both models in Figure 15.

Limitations and future work. Like all works dealing with microscale appearance, our results depend on the underlying model for the microfacets. Our method is thus inherently limited by the $\mathrm{V}$-grooves model, given our kaleidoscopic analysis of light transport. While this allows us a closed-form solution for multiple scattering, it imposes limitations in terms of physical realism at grazing angles, as well as discontinuities in the BRDF. Moreover, as described before, the particular correlation of V-grooves creates a caustic behavior, resulting into a specular peak even for high roughness; this is partially attenuated with the additional degree of freedom of nonsymmetric V-grooves. Reducing the correlation by introducing non-parallel V-grooves should eventually eliminate the singularity. Handling refraction with our model would in principle require a new kaleidoscopic model to account for the change of the ray's direction. Moreover, the splitting behavior of Fresnel equations in dielectrics might require several iterations of the model to account for the reflected and refracted rays. Finally, refraction might break the implicit locality of reflection inside the V-groove, since a refracted ray could potentially interact with several grooves before leaving the microsurface. 


\section{ACKNOWLEDGMENTS}

We want to thank Julio Marco for his help with figures, Ibón Guillén for discussions, and the members of the Graphics and Imaging Lab for proofreading. Min H. Kim acknowledges Korea NRF grants (2016R1A2B2013031, 2013M3A6A6073718) and additional support by KOCCA in MCST of Korea, Cross-Ministry Giga KOREA Project (GK17P0200), Samsung Electronics (SRFC-IT1402-02), and an ICT R\&D program of MSIT/IITP of Korea (2017-0-00072, 2016-0-00018). Diego Gutierrez and Adrian Jarabo are funded by the European Research Council (ERC) under the European Union's Horizon 2020 research and innovation program (CHAMELEON project, grant agreement No 682080), DARPA (project REVEAL), and the Spanish Ministerio de Economía y Competitividad (TIN2016-78753-P).

\section{APPENDICES}

\section{A COMPUTING THE PROJECTION FACTOR $\left\|\frac{\mathrm{d} \mathbf{s}}{\mathrm{d} \mathbf{o}}\right\|$}

To compute the projection factor, we compute the projection of partial steps, and multiply them to get the final factor. The transformation between $\mathbf{s}$ and $\mathbf{o}$ is thus divided in two steps: transformation between $\mathbf{s}$ and the halfway vector $\mathbf{h}$, and transformation between $\mathbf{h}$ and the outgoing direction $\mathbf{o :}$

$$
\left|\frac{\mathrm{d} \mathbf{s}}{\mathrm{d} \mathbf{o}}\right|=\left|\frac{\mathrm{d} \mathbf{s}}{\mathrm{d} \mathbf{h}} \frac{\mathrm{d} \mathbf{h}}{\mathrm{d} \mathbf{o}}\right|=\left|\frac{\mathrm{d} \mathbf{s}}{\mathrm{d} \mathbf{h}}\right|\left|\frac{\mathrm{d} \mathbf{h}}{\mathrm{d} \mathbf{o}}\right|,
$$

where $|\cdot|$ is the determinant of a matrix.

The halfway vector $\mathbf{h}=\left(\theta_{\mathbf{h}}, \phi_{\mathbf{h}}\right)$ and orientation $\mathbf{s}=\left(\theta_{\mathbf{s}}, \phi_{\mathbf{s}}\right)$ are on the $\mathbf{n}-\mathbf{s}$ plane, whose slope is computed by Equation (5):

$$
\begin{gathered}
\phi_{\mathbf{h}}=\phi_{\mathbf{s}}, \\
\theta_{\mathbf{h}}=\left((-1)^{k}\left(\theta_{i}+\pi-k\left(\pi-2 \theta_{\mathbf{s}}\right)\right)+\theta_{\mathbf{i}}\right) / 2 .
\end{gathered}
$$

The projection between $\mathbf{s}$ and $\mathbf{h}$ is:

$$
\begin{gathered}
\left\|\frac{\mathrm{d} \mathbf{s}}{\mathrm{dh}}\right\|= \\
=\frac{\sin \theta_{\mathbf{s}}}{\sin \theta_{\mathbf{h}}}\left\|\frac{\partial\left(\theta_{\mathbf{s}}, \phi_{\mathbf{s}}\right)}{\partial\left(\theta_{\mathbf{h}}, \phi_{\mathbf{h}}\right)}\right\|=\frac{\sin \theta_{\mathbf{s}}}{\sin \theta_{\mathbf{h}}}\left\|\begin{array}{cc}
\frac{\partial \theta_{\mathbf{s}}}{\partial \theta_{\mathbf{h}}} & \frac{\partial \theta_{\mathbf{s}}}{\partial \phi_{\mathbf{h}}} \\
\frac{\partial \phi_{\mathbf{s}}}{\partial \theta_{\mathbf{h}}} & \frac{\partial \phi_{\mathbf{s}}}{\partial \phi_{\mathbf{h}}}
\end{array}\right\| \\
=\frac{\sin \theta_{\mathbf{s}}}{\sin \theta_{\mathbf{h}}}\left\|\begin{array}{cc} 
\pm \frac{1}{k} & \frac{\partial \theta_{\mathbf{s}}}{\partial \phi_{\mathbf{h}}} \\
0 & 1
\end{array}\right\|=\frac{\sin \theta_{\mathbf{s}}}{k \sin \theta_{\mathbf{h}}} .
\end{gathered}
$$

For easy computation, we compute the projection factor in ipolar coordinates, where the incident direction $\mathbf{i}$ is a polar axis. The symbol ' denotes i-polar coordinates. We compute the projection factor of the transformation between $\mathbf{h}^{\prime}=\left(\theta_{\mathbf{h}^{\prime}}, \phi_{\mathbf{h}^{\prime}}\right)$ and orientation $\mathbf{o}=\left(\theta_{\mathbf{o}^{\prime}}, \phi_{\mathbf{o}^{\prime}}\right)=\left(2 \theta_{\mathbf{h}^{\prime}}, \phi_{\mathbf{h}^{\prime}}\right):$

$$
\left\|\frac{\mathrm{d} \mathbf{h}^{\prime}}{\mathrm{d} \mathbf{o}^{\prime}}\right\|=\frac{\sin \theta_{\mathbf{h}^{\prime}}}{\sin \theta_{\mathbf{o}^{\prime}}}\left\|\frac{\partial\left(\theta_{\mathbf{h}^{\prime}}, \phi_{\mathbf{h}^{\prime}}\right)}{\partial\left(\theta_{\mathbf{o}^{\prime}}, \phi_{\mathbf{o}^{\prime}}\right)}\right\|=\frac{\sin \theta_{\mathbf{h}^{\prime}}}{\sin 2 \theta_{\mathbf{h}^{\prime}}}\left|\begin{array}{cc}
0.5 & 0 \\
0 & 1
\end{array}\right|=\frac{1}{4 \cos \theta_{\mathbf{h}}^{\prime}} .
$$

By substituting Equations (28) and (29) into Equation (26), the projection factor of the transformation between $\mathbf{s}$ and $\mathbf{o}$ is computed as

$$
\left\|\frac{\mathrm{d} \mathbf{s}}{\mathrm{d} \mathbf{o}}\right\|=\left\|\frac{\mathrm{d} \mathbf{s}^{\prime}}{\mathrm{d} \mathbf{o}^{\prime}}\right\|=\left\|\frac{\mathrm{d} \mathbf{s}^{\prime}}{\mathrm{d} \mathbf{s}} \frac{\mathrm{d} \mathbf{s}}{\mathrm{dh}} \frac{\mathrm{d} \mathbf{h}}{\mathrm{d} \mathbf{h}^{\prime}} \frac{\mathrm{d} \mathbf{h}^{\prime}}{\mathrm{d} \mathbf{o}^{\prime}}\right\|=\left\|\frac{\mathrm{d} \mathbf{s}}{\mathrm{d} \mathbf{h}}\right\|\left\|\frac{\mathrm{d} \mathbf{h}^{\prime}}{\mathrm{d} \mathbf{o}^{\prime}}\right\|=\frac{\sin \theta_{\mathbf{s}}}{k \sin \theta_{\mathbf{h}}} \frac{1}{4 \cos \theta_{\mathbf{h}}^{\prime}} .
$$

Note that the deformation factor of the transform between $\mathbf{i}$-polar coordinate and $\mathbf{n}$-polar coordinate $\left\|\frac{\mathrm{da}}{\mathrm{d} \mathbf{a}^{\prime}}\right\|$ is 1 .

\section{B PROOF FOR UNIQUENESS OF POINT $\mathbf{k}$}

For a given symmetric V-groove with angle $\theta_{v}$, we set the origin of our coordinate system as shown in Figure 4a. The left and right upper-points of the V-groove are $\mathbf{a}$ and $\mathbf{b}$, with light coming from the incoming direction $\mathbf{i}$. Let us define a circle $C$ passing through $\mathbf{a}$ and $\mathbf{b}$, with origin at $\bar{\omega}$. If we trace two lines from $\mathbf{a}$ and $\mathbf{b}$ in the incoming direction $\mathbf{i}$ they intersect the circle $C$ at points $\mathbf{a}^{\prime}$ and $\mathbf{b}^{\prime}$, respectively. We denote $\theta_{x}$ as the angle between $\bar{\omega}$ and an arbitrary point $x$ and $\mathbf{n}$ in an counter-clockwise manner. In the following, we derive two lemmas to demonstrate our theorem.

Lemma B.1. The angle of the arc between $\boldsymbol{a}^{\prime}$ and $\boldsymbol{b}^{\prime}$ is $\theta_{v}$.

Proof. Thanks to the circle property, the arc between $\mathbf{a}^{\prime}$ and $\mathbf{b}^{\prime}$ is line symmetric to the arc between $\mathbf{a}$ and $\mathbf{b}$ along line $\left(\mathbf{i}_{\perp}, \bar{\omega}\right)$. Therefore the angle of arc between $\mathbf{a}^{\prime}$ and $\mathbf{b}^{\prime}$ has the same angle as the arc between $\mathbf{a}$ and $\mathbf{b}$, due to their symmetric relation.

As we generate virtual grooves by reflecting them along the leftmost edges, we generate several new groove edges. Let point $P_{k}$ be the end point of the $k$-th edge.

Lemma B.2. All reflected $V$-grooves have the same angle $\theta_{v}$.

Proof. For every $i$-th reflection, the $(i+1)$-th reflected V-groove has the same angle as the $i$-th V-groove due to line symmetry. By induction, every reflected $\mathrm{V}$-groove angle has the same angle as the 1st V-groove $\theta_{v}$.

Theorem B.3. Only one edge point $P_{k}$ exists so that $\theta_{P_{k}} \in\left[\theta_{\boldsymbol{a}^{\prime}}, \theta_{\boldsymbol{b}^{\prime}}\right)$.

Proof. To prove the existence of at least one $\theta_{P_{k}} \in\left[\theta_{\mathbf{a}^{\prime}}, \theta_{\mathbf{b}^{\prime}}\right)$, let us assume that $P_{k+1}$ is the closest point to $\mathbf{b}^{\prime}$, and that $\theta_{P_{k+1}} \geq \theta_{\mathbf{a}^{\prime}}$. By reductio ad absurdum, let us assume that there is no V-groove edge $k$ so that $\theta_{P_{k}} \in\left[\theta_{\mathbf{a}^{\prime}}, \theta_{\mathbf{b}^{\prime}}\right)$; therefore, since $\theta_{P_{k}}<\theta_{P_{k+1}}$, that means that $\theta_{P_{k}}<\theta_{\mathbf{a}^{\prime}}$. By Lemma 1, it means that the angle of the $k$-th V-groove is larger than the 1 st V-groove's angle $\theta_{v}$, which contradicts Lemma 2:

$$
\begin{aligned}
\theta_{P_{k}}<\theta_{\mathbf{a}^{\prime}}<\theta_{\mathbf{b}^{\prime}} \leqslant \theta_{P_{k+1}} \Rightarrow \theta_{v}=\theta_{\mathbf{b}^{\prime}}-\theta_{\mathbf{a}^{\prime}} & \leqslant \theta_{P_{k+1}}-\theta_{\mathbf{a}^{\prime}} \\
& <\theta_{P_{k+1}}-\theta_{P_{k}}=\theta_{v}, \\
\Rightarrow \perp &
\end{aligned}
$$

Therefore, there is at least one point $\mathbf{k}$ on the $\operatorname{arc}$ between $\mathbf{a}^{\prime}$ and $\mathbf{b}^{\prime}$, including $\mathbf{a}^{\prime}$.

In order to prove uniqueness, let us suppose that there are two edge points $P_{k-1}$ and $P_{k}$ on the arc between $\mathbf{a}^{\prime}$ and $\mathbf{b}^{\prime}$. Then the angle of the $k$-th $\mathrm{V}$-groove needs to be smaller than the $\theta_{v}$, which contradicts Lemma 2:

$$
\begin{aligned}
\theta_{\mathbf{a}^{\prime}} \leqslant \theta_{P_{k}-1}<\theta_{P_{k}}<\theta_{\mathbf{b}^{\prime}} \Rightarrow \theta_{v}=\theta_{\mathbf{b}^{\prime}}-\theta_{\mathbf{a}^{\prime}} & >\theta_{P_{k}}-\theta_{\mathbf{a}^{\prime}} \\
& \geqslant \theta_{P_{k}}-\theta_{P_{k-1}}=\theta_{v}, \\
\Rightarrow \perp . &
\end{aligned}
$$

Therefore, there is only one $\mathbf{k}$ on the $\operatorname{arc}$ between $\mathbf{a}^{\prime}$ and $\mathbf{b}^{\prime}$, including point $\mathbf{a}^{\prime}$.

ACM Trans. Graph., Vol. 37, No. 6, Article 275. Publication date: November 2018. 


\section{DEFINING $P(\theta)$}

Here we include the probability distributions $P\left(\theta_{r}\right)$ defined in the nonsymmetric V-groove model, that ensure that $\mathbf{s}_{r}$ follows a given probability distribution $D(\mathbf{s})$. As described in Section 5.2, assuming independence between $\theta_{r}$ and $\theta_{l}$, then $P\left(\theta_{r}\right)$ follows Equation (20). In the following, we describe $P\left(\theta_{r}\right)$ for Beckmann, GGX, and Phong normal distribution functions $\left(D_{\mathrm{B}}(\mathbf{s}), D_{\mathrm{G}}(\mathbf{s})\right.$, and $D_{\mathrm{P}}(\mathbf{s})$ respectively).

Beckmann NDF:

$$
P_{\mathrm{B}}\left(\theta_{r}\right)=\frac{\alpha}{2 \sqrt{\pi}} D_{\mathrm{B}}\left(\mathbf{s}_{r}\right) \sin ^{2} \theta_{r} .
$$

GGX NDF:

$$
P_{\mathrm{G}}\left(\theta_{r}\right)=\frac{\alpha}{4} D_{\mathrm{G}}\left(\mathbf{s}_{r}\right) \sin ^{2} \theta_{r}
$$

Phong NDF:

$$
P_{\mathrm{P}}\left(\theta_{r}\right)=\frac{\alpha_{p}+2}{8 \sqrt{\pi}} \frac{\gamma\left(\frac{\alpha_{p}+1}{2}\right) \gamma\left(\frac{\alpha_{p}+3}{2}\right)}{\gamma\left(\frac{\alpha_{p}+4}{2}\right)} D_{\mathrm{P}}\left(\mathbf{s}_{r}\right) \sin ^{2} \theta_{r} .
$$

Unfortunately, none of these distributions have an analytical form for the inverted $\mathrm{CDF}$.

\section{REFERENCES}

M. Ashikmin, S. Premoze, and P. Shirley. 2000. A Microfacet-based BRDF Generator. In SIGGRAPH. 65-74.

S.-H. Baek, D. S. Jeon, X. Tong, and M. H. Kim. 2018. Simultaneous Acquisition of Polarimetric SVBRDF and Normals. ACM Trans. Graph. 37, 6 (2018).

P. Beckmann and A. Spizzichino. 1963. The scattering of electromagnetic waves from rough surfaces. Int Ser. Monographs. Electomagn Waves 4 (1963).

C. Bosch, X. Pueyo, S. Merillou, and D. Ghazanfarpour. 2004. A Physically Based Model for Rendering Realistic Scratches. Comput. Graph. Forum 23, 3 (2004), 361-370.

C. Bourlier and G. Berginc. 2004. Multiple scattering in the high-frequency limit with second-order shadowing function from $2 \mathrm{D}$ anisotropic rough dielectric surfaces: $\mathrm{I}$ Theoretical study. Wave. Random Media 14, 3 (2004), 253-276.

C. Bourlier, G. Berginc, and J. Saillard. 2002. Monostatic and bistatic statistical shadowing functions from a one-dimensional stationary randomly rough surface: II Multiple scattering. Wave. Random Media 12, 2 (2002), 175-200.

R. L. Cook and K. E. Torrance. 1982. A Reflectance Model for Computer Graphics. ACM Trans. Graph. 1, 1 (1982), 7-24.

Z. Dong, B. Walter, S. Marschner, and D. P. Greenberg. 2015. Predicting appearance from measured microgeometry of metal surfaces. ACM Trans. Graph. 35, 1 (2015), 9.

J. Dupuy, E. Heitz, and E. d'Eon. 2016. Additional Progress Towards the Unification of Microfacet and Microflake Theories. In Proc. Eurograph. Symp. Rendering. 55-63.

B. W. Hapke. 1963. A theoretical photometric function for the lunar surface. F. Geophy. Re. 68, 15 (1963), 4571-4586.

E. Heitz. 2014. Understanding the Masking-Shadowing Function in Microfacet-Based BRDFs. 7. Comput. Graph. Techn. 3, 2 (2014), 48-107.

E. Heitz and E. d'Eon. 2014. Importance Sampling Microfacet-Based BSDFs using the Distribution of Visible Normals. Comput. Graph. Forum 33, 4 (2014), 103-112.

E. Heitz, J. Hanika, E. d'Eon, and C. Dachsbacher. 2016. Multiple-scattering Microfacet BSDFs with the Smith Model. ACM Trans. Graph. 35, 4 (2016), 58:1-58:14.

N. Holzschuch and R. Pacanowski. 2017. A two-scale microfacet reflectance model combining reflection and diffraction. ACM Trans. Graph. 36, 4 (2017), 66.

W. Jakob. 2010. Mitsuba renderer. http://www.mitsuba-renderer.org.

W. Jakob, E. d'Eon, O. Jakob, and S. Marschner. 2014. A Comprehensive Framework for Rendering Layered Materials. ACM Trans. Graph. 33, 4 (2014), 118:1-118:14.

C. Kelemen and L. Szirmay-Kalos. 2001. A Microfacet Based Coupled Specular-Matte BRDF Model with Importance Sampling. In Proc. Eurograph. Short Presentations, Vol. 2. 4.

J. J. Koenderink, A. J. Van Doorn, K. J. Dana, and S. Nayar. 1999. Bidirectional reflection distribution function of thoroughly pitted surfaces. Int. F. Comput. Vis. 31, 2-3 (1999), 129-144.

C. Kulla and A. Conty. 2017. Revisiting Physically Based Shading at Imageworks. In Physically Based Shading in Theory and Practice, SIGGRAPH 2017 Courses.

S. Merillou, J.M. Dischler, and D. Ghazanfarpour. 2001. Surface scratches: measuring, modeling and rendering. Vis. Comput. 17, 1 (2001), 30-45.

G. Nam, J. H. Lee, D. Gutierrez, and M. H. Kim. 2018. Practical SVBRDF Acquisition of 3D Objects with Unstructured Flash Photography. ACM Trans. Graph. 37, 6 (2018).
G. Nam, J. H. Lee, H. Wu, D. Gutierrez, and M. H. Kim. 2016. Simultaneous Acquisition of Microscale Reflectance and Normals. ACM Trans. Graph. 35, 6 (2016), 185:1-185:11.

M. Oren and S. K. Nayar. 1994. Generalization of Lambert's Reflectance Model. In SIGGRAPH. 239-246.

P. Poulin and A. Fournier. 1990. A Model for Anisotropic Reflection. SIGGRAPH Comput. Graph. 24, 4 (1990), 273-282.

B. Raymond, G. Guennebaud, and P. Barla. 2016. Multi-scale rendering of scratched materials using a structured SV-BRDF model. ACM Trans. Graph. 35, 4 (2016), 57.

S. Rusinkiewicz. 1998. A New Change of Variables for Efficient BRDF Representation. In Rendering Techniques '98 (Proceedings of Eurographics Rendering Workshop '98), G. Drettakis and N. Max (Eds.). Springer Wien, 11-22.

D. Saint-Pierre, R. Deeb, D. Muselet, L. Simonot, and M. Hébert. 2018. Light interreflections and shadowing effects in a Lambertian V-cavity under diffuse illumination. 7 . Electron. Imag. (2018).

B. Smith. 1967. Geometrical shadowing of a random rough surface. IEEE Trans. Antennas Propag. 15, 5 (1967), 668-671.

K. E. Torrance and E. M. Sparrow. 1967. Theory for Off-Specular Reflection From Roughened Surfaces. 7. Opt. Soc. Am. 57, 9 (1967), 1105-1114.

B. Walter, S. R. Marschner, H. Li, and K. E. Torrance. 2007. Microfacet Models for Refraction Through Rough Surfaces. In Proc. Eurograph. Symp. Rendering. 195-206.

R. B. Zipin. 1966. The apparent thermal radiation properties of an isothermal V-groove with specularly reflecting walls. F. Res. NBS C 70 (1966), 275-280. 